\title{
Review Article \\ Status of the GERDA Experiment at the Laboratori Nazionali del Gran Sasso
}

\author{
R. Brugnera ${ }^{1,2}$ and A. Garfagnini ${ }^{1,2}$ \\ ${ }^{1}$ Dipartimento di Fisica e Astronomia, Università di Padova, Via Marzolo 8, 35131 Padova, Italy \\ ${ }^{2}$ Istituto Nazionale di Fisica Nucleare, Sezione di Padova, Via Marzolo 8, 35131 Padova, Italy \\ Correspondence should be addressed to R. Brugnera; riccardo.brugnera@pd.infn.it
}

Received 30 July 2013; Accepted 7 October 2013

Academic Editor: Vincenzo Flaminio

Copyright (c) 2013 R. Brugnera and A. Garfagnini. This is an open access article distributed under the Creative Commons Attribution License, which permits unrestricted use, distribution, and reproduction in any medium, provided the original work is properly cited.

\begin{abstract}
The Germanium Detector Array (GERDA) is a low background experiment at the Laboratori Nazionali del Gran Sasso (LNGS) of the INFN designed to search for the rare neutrinoless double beta decay $(0 \nu \beta \beta)$ of ${ }^{76} \mathrm{Ge}$. In its first phase, high purity germanium diodes inherited from the former Heidelberg-Moscow and IGEX experiments are operated "bare" and immersed in liquid argon, with an overall background environment of $10^{-2} \mathrm{cts} /(\mathrm{keV} \cdot \mathrm{kg} \cdot \mathrm{yr})$, a factor of ten better than its predecessors. Measurements on twoneutrino double beta decay $(2 \nu \beta \beta)$ giving $T_{1 / 2}^{2 \nu}=(1.88 \pm 0.10) \times 10^{21} \mathrm{yr}$ and recently published background model and pulse shape performances of the detectors are discussed in the paper. A new result on $0 \nu \beta \beta$ has been recently published with a half-life limit on $0 \nu \beta \beta$ decay $T_{1 / 2}^{0 \nu}>2.1 \times 10^{25} \mathrm{yr}(90 \%$ C.L.). A second phase of the experiment is scheduled to start during the year 2014 , after a major upgrade shutdown. Thanks to the increased detector mass with new designed diodes and to the introduction of liquid argon instrumentation techniques, the experiment aims to reduce further the expected background to about $10^{-3} \mathrm{cts} /(\mathrm{keV} \cdot \mathrm{kg} \cdot \mathrm{yr})$ and to improve the $0 \nu \beta \beta$ sensitivity to about $T_{1 / 2}^{0 v}>1.5 \times 10^{26} \mathrm{yr}$ (90\% C.L.).
\end{abstract}

\section{Introduction}

Double beta decay is the simultaneous beta decay of two neutrons in a nucleus. It is a second-order weak process, predicted by the standard model: $(A, Z) \rightarrow(A, Z+2)+2 e^{-}+$ $2 \bar{v}_{e}$. The process $(2 v \beta \beta)$ has been experimentally observed in even-even nuclei and can be detected only when its single beta decay is energetically forbidden or strongly suppressed because of a large change of spin. Typical half-lives are very large and lie between $7 \times 10^{18} \mathrm{yr}$ and $2 \times 10^{24} \mathrm{yr}$ [1-6]. Extensions of the standard model $(\mathrm{SM})$ predict that also neutrinoless double beta decay, $(A, Z) \rightarrow(A, Z+2)+2 e^{-}$, could occur $(0 \nu \beta \beta)$. Its observation implies that the lepton number is violated by two units. It would prove that neutrinos have a Majorana mass component, and consequently they are their own antiparticle. With the further assumption that $0 \nu \beta \beta$ decay is mediated by the exchange of light Majorana neutrinos, an effective neutrino mass can be evaluated through the use of predictions for the nuclear matrix element and the phase space factor. For recent reviews on $0 \nu \beta \beta$, see [7-10].
The Gerda [11] experiment in Europe and Majorana $[12,13]$ in the USA, at different stages of development and using different experimental approaches, are the current state-of-the-art experiments to search for $0 \nu \beta \beta$ in ${ }^{76} \mathrm{Ge}$ using germanium detectors. The process has a clear signature with a monoenergetic line in the observed energy spectrum at $Q_{\beta \beta}=2039.061 \pm 0.007 \mathrm{keV}$ [14], corresponding to the sum of the two electrons energies. Two previous experiments, Heidelberg-Moscow (HDM) and IgEx, have studied double beta decay in germanium and set limits on the $0 \nu \beta \beta$ half-life: $T_{1 / 2}^{0 \nu}>1.9 \times 10^{25} \mathrm{yr}[15]$ and $T_{1 / 2}^{0 \nu}>1.6 \times 10^{25} \mathrm{yr}[16-18]$, respectively. Part of the HDM collaboration claimed evidence for a peak at $Q_{\beta \beta}$ which corresponds to a half-life central value of $T_{1 / 2}^{0 \nu}=1.19_{-0.23}^{+0.37} \times 10^{25} \mathrm{yr}[19]$. The result was later refined with pulse shape discrimination (PSD) analysis techniques giving a half-life $T_{1 / 2}^{0 v}=2.23_{-0.31}^{+0.44} \times 10^{25} \mathrm{yr}$ [20]. Due to various inconsistencies in the analysis pointed out in [7], only the first claim will be considered in the following.

The data taking of the GERDA experiment has been organized in two phases. During Phase I, the experiment aims 


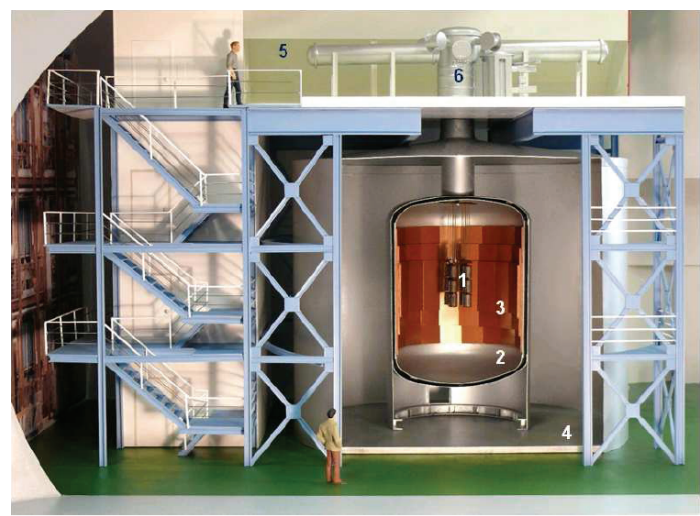

FIgURe 1: Artist view of the Gerda experiment. The following components are labeled in the picture: (1) the germanium array strings (not to scale), (2) the LAr cryostat, (3) the internal copper shield, (4) the instrumented water tank, and (5) the clean room with the lock insertion system (6). From [11].

to scrutinize the HDM claim, using high purity germanium detectors coming from the HDM and IGEx collaborations (about $18 \mathrm{~kg}$ of total mass) and operating in a environment with a lower background. The sensitivity will be improved by an order of magnitude during the Phase II with the help of an additional set of new detectors (increasing the mass by about $20 \mathrm{~kg}$ ) and a higher background suppression thanks to liquid argon (LAr) instrumentation and enhanced PSD of the new detectors.

In the following sections, after an introduction to the experimental setup, the Phase I results will be reviewed: the first $2 \nu \beta \beta$ half-life measurement, a detailed study of the GERDA background decomposition model, and the detector performances with respect to PSD techniques. Finally, the recent $0 \nu \beta \beta$ half-life measurement is discussed and the implications of the limit with respect to those determined in ${ }^{76} \mathrm{Ge}$ $[19]$ and with ${ }^{136}$ Xe based experiments $[21,22]$ are illustrated.

\section{The GERDA Detector}

The GERDA [11] experiment, at the Laboratori Nazionali del Gran Sasso (LNGS) of INFN, operates bare germanium diodes made of isotopically modified material, enriched to about $86 \%$ in ${ }^{76} \mathrm{Ge}$, in a LAr cryogenic bath, without their usual vacuum cryostats. The experiment aims to pursue very low backgrounds thanks to ultrapure shielding against environmental radiation. An artist view of the detector is given in Figure 1. The germanium detectors are suspended in strings into the cryostat. The $64 \mathrm{~m}^{3}$ of LAr are used both as a coolant and shield. The stainless steel cryostat vessel is covered, from the inside, with copper lining to reduce gamma radiation from the cryostat walls. The vessel is surrounded by a large tank filled with high purity water $\left(590 \mathrm{~m}^{3}\right)$ which further shields the inner volumes from the experimental hall radiation (absorbing $\gamma$ s and moderating neutrons). Moreover it provides a sensitive medium for the muon system which operates as a Cherenkov muon veto.
On top of the GERDA building, just above the cryostat neck, a ISO class 7 clean room has been installed and is used as a working environment within which a nitrogen fluxed glove box is used to manipulate the detectors before inserting them into the cryostat. The detectors are arranged in strings, each supporting three semicoaxial or five broad energy germanium (BEGe) detectors and are lowered into the cryostat through a two-arm lock which separates the clean room from the cryostat. The detector strings are surrounded by a $60 \mu \mathrm{m}$ thick copper cylinder (called minishroud) with dimensions such that the detector outer surface is placed at few $\mathrm{mm}$ from the copper walls. A larger enclosure-a $30 \mu \mathrm{m}$ thick copper cylinder-with a $75 \mathrm{~cm}$ diameter is built around all detector strings.

All detectors are read out with charge sensitive preamplifiers (called CC2 [23]) operated in LAr at about $30 \mathrm{~cm}$ for the top of the detector array. The CC2, located inside a copper box providing electromagnetic shielding, drives $20 \mathrm{~m}$ long signal cables from the cold LAr region to the room temperature DAQ electronics through custom made feedthroughs. The analog signals are digitized by $100 \mathrm{MHz}$ FADCs. The raw data, saved in binary format, is converted to a standardized format defined by MGDO (MAJORANA-GERDA Data Objects) [24], a software library jointly developed by the GERDA and MAJORANA collaborations that contains general-purpose interfaces and analysis tools supporting digital processing of experimental or simulated signals. The MGDO data objects are stored as Root files [25], named Tier1. The Gelatio software framework [26] provides independent and customizable modules which are applied to the TIER1 waveforms producing several observables (pulse amplitude, rise time, average baseline, etc.) which are stored in new Rоoт files (TIER2) and are used for both data and Monte Carlo.

Eight reprocessed semicoaxial germanium detectors, inherited from the previous HDM and IGEX experiments, were deployed on November 9, 2011, together with three detectors with natural isotopic abundance. In July 2012, two of the semicoaxial diodes with natural isotopic abundance were replaced by five enriched BEGe detectors. The latter follow the Phase II design of GERDA and were recently characterized by the collaboration with the HEROICA [27] infrastructure at HADES (Belgium).

Further information on the detectors properties used in Gerda Phase I are available here [28], while details on the detector design, construction, and first results on the commissioning phase can be found here [11].

2.1. Germanium Detectors. In Gerda two types of germanium detectors are used: semicoaxial and BEGe. A sketch of both reporting their typical geometrical dimensions, electrodes, grooves, and weighting potentials [29] is shown in Figure 2. The semicoaxial germanium detectors used in Phase I are standard p-type detectors, with a $\mathrm{n}^{+}$conductive lithium layer $(\sim 1 \mathrm{~mm})$ separated from the boron implanted $\mathrm{p}^{+}$contact by a groove. In normal DC coupled readout, the $\mathrm{p}^{+}$surface $(\sim 1 \mu \mathrm{m})$ is connected to a charge sensitive amplifier and the $\mathrm{n}^{+}$surface is biased with up to $+4600 \mathrm{~V}$. For Phase II new detectors will be used of BEGe type. The detector has a cylindrical shape, a small boron-implanted $\mathrm{p}^{+}$electrode 


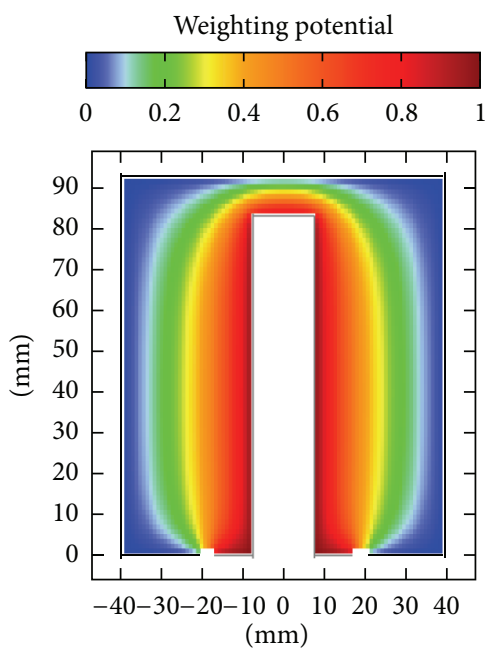

(a)

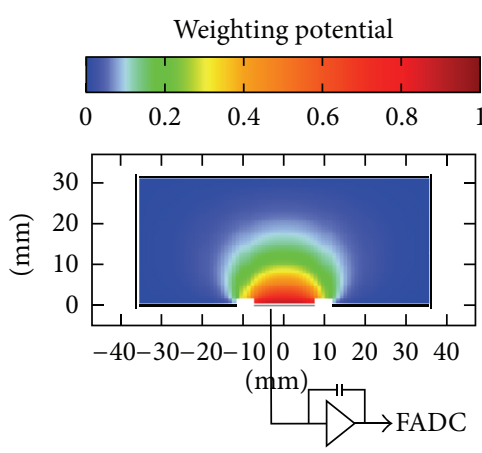

(b)

Figure 2: Cross-section of a semicoaxial detector (a) and a BEGe detector (b). The $\mathrm{n}^{+}$electrode is drawn in black and the $\mathrm{p}^{+}$electrode in gray; the thicknesses are not to scale. The two electrodes are separated by an insulating groove. The weighting potential [29] inside the detectors is indicated through a color map. For the BEGE also the readout with a charge sensitive amplifier is shown. From [32].

is placed on one of the flat surfaces. The lithium-diffused $\mathrm{n}^{+}$electrode, covers the rest of the outer surface and it is separated from the $\mathrm{p}^{+}$electrode by a circular groove. The positive bias is applied to the $\mathrm{n}^{+}$electrode, while the $\mathrm{p}^{+}$ electrode (signal read-out electrode) is grounded. The main characteristic of this detector is the small area $\mathrm{p}^{+}$electrode. This implies a lower capacitive noise and so an improved energy resolution in a wide range with respect to the standard semicoaxial detectors. Moreover this geometrical configuration in conjunction with an ad hoc impurities profile inside the detector permits to obtain enhanced PSD capabilities.

2.2. Data Taking. The data taking started on November 9, 2011, and finished on May 21, 2013. A total exposure of $21.60 \mathrm{~kg} \cdot \mathrm{yr}$ was collected, subdivided into $19.20 \mathrm{~kg} \cdot \mathrm{yr}$ from the semicoaxial detectors and $2.40 \mathrm{~kg} \cdot \mathrm{yr}$ from the BEGe detectors. Table 1 shows the different exposures used in the analyses explained in the following sections, while Table 2 illustrates how the exposure, for the different analyses, is then
TABLE 1: List of the various analyses illustrated in the paper with the corresponding exposures and data taking time periods.

\begin{tabular}{lccc}
\hline \multicolumn{2}{c}{ Period } & $\begin{array}{c}\text { Exposure } \\
\mathrm{kg} \cdot \mathrm{yr}\end{array}$ & Analysis \\
\hline From & To & 5.04 & $2 \nu \beta \beta$ \\
$09 / 11 / 2011$ & $21 / 03 / 2012$ & 18.50 & Background decomposition \\
$09 / 11 / 2011$ & $3 / 03 / 2013$ & PSD analysis \\
$09 / 11 / 2011$ & $21 / 05 / 2013$ & 21.60 & $0 \nu \beta \beta$ \\
\hline
\end{tabular}

TABLE 2: Data sets, the detectors used and their exposure are listed for the data used for the background decomposition, PSD and $0 \nu \beta \beta$ analyses.

\begin{tabular}{|c|c|c|c|}
\hline \multirow{3}{*}{ Data set } & \multirow{3}{*}{ Detectors } & \multicolumn{2}{|c|}{ Exposure } \\
\hline & & $\begin{array}{c}\text { Background } \\
\text { decomposition }\end{array}$ & $\begin{array}{l}0 \nu \beta \beta \text { analysis } \\
\text { PSD analysis }\end{array}$ \\
\hline & & \multicolumn{2}{|c|}{$\mathrm{kg} \cdot \mathrm{yr}$} \\
\hline$S U M-\operatorname{coa} x$ & $\begin{array}{l}\text { all enriched } \\
\text { semicoaxial }\end{array}$ & 16.70 & 19.20 \\
\hline GOLD-coax & $\begin{array}{l}\text { all enriched } \\
\text { semicoaxial }\end{array}$ & 15.40 & 17.90 \\
\hline SILVER-coax & $\begin{array}{l}\text { all enriched } \\
\text { semicoaxial }\end{array}$ & 1.30 & 1.30 \\
\hline SUM-bege & all enriched BEGe & 1.80 & 2.40 \\
\hline GOLD-nat & GTF 112 & 3.13 & 3.98 \\
\hline
\end{tabular}

subdivided into various data sets. The reason to divide the exposure of the semicoaxial detectors into two different data sets (GOLD-coax and SILVER-coax) is due to the fact that for approximately 30 days after the BEGe detectors deployment the background counting rate increased a lot. All the quoted numbers for the exposures are calculated from the total detector mass. In the analyses the isotopic abundance of the ${ }^{76} \mathrm{Ge}$ and the active mass are properly taken into account. The abundances ranges vary from $86 \%$ to $88 \%$ while the active volume fractions, that is, the ratio between active detector mass and total detector mass, from $83 \%$ to $92 \%$.

The energy scale of the individual detectors was determined with ${ }^{228} \mathrm{Th}$ sources once every one or two weeks. The energy resolution was stable over the entire data acquisition period. At the $Q_{\beta \beta}$ point, the exposure-averaged values are $(4.8 \pm 0.2) \mathrm{keV}$ for semicoaxial and $(3.2 \pm 0.2) \mathrm{keV}$ for BEGe, respectively. The differences between reconstructed peak positions and the ones from the calibration curves are smaller than $0.3 \mathrm{keV}$.

2.3. Background Sources. In the experiments dedicated to the search of $0 \nu \beta \beta$ events the level of the background is given in terms of the so-called background index (BI), that is, the background counts per keV, per $\mathrm{kg}$, and per year in the region of interest (ROI) around $Q_{\beta \beta}$. In order to achieve its sensitivity goals, in Phase I GERDA has to confine the BI at the level of $10^{-2} \mathrm{cts} /(\mathrm{keV} \cdot \mathrm{kg} \cdot \mathrm{yr})$ and down to $10^{-3} \mathrm{cts} /(\mathrm{keV} \cdot \mathrm{kg} \cdot \mathrm{yr})$ in Phase II. 
The sources of background can be classified by the location of the radioactive isotope: backgrounds internal to the germanium diode and external backgrounds.

The backgrounds internal to the germanium diode are generated during the diode production above ground. There the germanium is exposed to hadronic radiation, especially neutrons. These cause spallation in the material and hence a variety of isotopes are produced. Most important for the neutrinoless double beta decay are the decays of ${ }^{68} \mathrm{Ge}$ and ${ }^{60} \mathrm{Co}$ since $Q$ values above $Q_{\beta \beta}=2039 \mathrm{keV}$ occur in the chain, and the lifetimes are in the range of years. The cosmogenic production of ${ }^{60} \mathrm{Co}$ in a germanium detector is about 4 atoms/ $(\mathrm{kg} \cdot \mathrm{d})$ above ground [30], while the production rate of ${ }^{68} \mathrm{Ge}$ in ${ }^{76} \mathrm{Ge}$ is about 1 atom/(kg.d) [30]. The final internal background contamination for the semicoaxial detector was estimated to be $10^{-2} \mathrm{cts} /(\mathrm{keV} \cdot \mathrm{kg} \cdot \mathrm{yr})$ mainly due to ${ }^{60} \mathrm{Co}$ activity. For the BEGe detectors all the various steps from the enrichment of the material to the diode production were realized minimizing the time exposure on the surface.

The external backgrounds consist of photons from primordial decay chains, neutrons, and muon induced background. The cryostat and the water tank were designed in order to suppress the contribution from $2.615 \mathrm{MeV}$ photons from ${ }^{208} \mathrm{Tl}$ decays (coming from the rock in LNGS Hall A and cryostat material), from neutrons (coming from spontaneous fission mainly by the ${ }^{238} \mathrm{U}$ isotope, from $(\alpha, n)$ reactions in the concrete and the rock and from muon interactions). For all these background components the $\mathrm{BI}$ is reduced to a level of $10^{-4} \mathrm{cts} /(\mathrm{keV} \cdot \mathrm{kg} \cdot \mathrm{yr})$. The LAr contains the ${ }^{42} \mathrm{Ar}$ isotope, electrons from the ${ }^{42} \mathrm{~K}$ decays, the progeny of ${ }^{42} \mathrm{Ar}$, have energies up to $3.5 \mathrm{MeV}$, well above the $Q_{\beta \beta}$. Beta decays of ${ }^{39} \mathrm{Ar}$ have a $\mathrm{Q}$ value of $565 \mathrm{keV}$ and do not contribute to the background for neutrinoless double beta decay. In LAr also some ${ }^{222} \mathrm{Rn}$ impurities can be present. The experience of many low background experiments shows that surface contaminations are often larger than bulk activities; the main isotopes are ${ }^{232} \mathrm{Th}$ and ${ }^{210} \mathrm{~Pb}$. Another important source of contamination is the holder material of the diodes: simulations show that the fraction of decays depositing energy in the interval 2.0 to $2.8 \mathrm{MeV}$ is similar to the case of surface contaminations.

2.4. Background Rejection Techniques. In order to achieve the goal of one or two orders of magnitude reduction in backgrounds relative to previous experiments, a very careful selection of materials was made during Phase I. This selection was carried out by using state-of-the-art screening techniques: gamma ray spectroscopy with high purity germanium spectrometers in underground laboratories, gas counting with ultralow background proportional counters and mass spectrometry with inductively coupled plasma mass spectrometers (ICP-MS). To reduce the background further active techniques have to be used.

(i) Anticoincidence between different detectors in the setup: this method relies on the fact that $0 \nu \beta \beta$ events are single site events (SSE), while large part of the background can deposit its energy in many distant locations inside one detector and also in different detectors (multisite events, MSE).

(ii) PSD analysis: again, the nonlocalized energy deposition for background events is explored (for further details see Section 3.3).

(iii) Scintillation light detection: ionizing radiation that creates background signals in the diode with energies close to $Q_{\beta \beta}$ typically has energies greater than the one deposited in the germanium crystals. Part of this energy is dissipated in the LAr and is visible. The scintillation properties of LAr are well established: about 40,000 photons are emitted per $\mathrm{MeV}$ of deposited energy. Photons are emitted in the deexcitation of the $\mathrm{Ar}_{2}^{*}$ excimer with a wavelength of $128 \mathrm{~nm}$. These powerful techniques will be used during Phase II of the GERDA experiment.

\section{Results}

When all the detectors of Phase I were deployed into the LAr, the experiment strategy was to maximize the exposure under stable conditions. In parallel, an effort to understand the composition of the collected data and to develop methods to suppress a large fraction of background over signal $(0 \nu \beta \beta$ events) has been started. In the following sections, first the determination of the $2 \nu \beta \beta$ half-life [31] and afterwards the full spectrum background decomposition [28] will be described. After a discussion on the PSD methods [32] developed for background suppression, the first GERDA results on the $0 \nu \beta \beta$ process [33] will be reported.

3.1. Determination of the Half-Life of the $2 \nu \beta \beta$ Decay. The $2 \nu \beta \beta$ decay of atomic nuclei, with the simultaneous emission of two electrons and two antineutrinos, conserves lepton number and is allowed within the SM, regardless of the nature of the neutrino. It is characterized by an extremely low decay rate because it is a second-order process. So far it is the rarest decay ever observed in laboratory experiments. The measurement of the $2 v \beta \beta$ half-life $\left(T_{1 / 2}^{2 \nu}\right)$ is of some interest. Indeed it has been suggested [34-41] that, within the same model framework, the evaluation of the nuclear matrix element for the $2 \nu \beta \beta$ decay $\left(M_{2 v}\right)$ from $T_{1 / 2}^{2 \nu}$ measurements could set some constraints on $M_{0 v}$. Moreover, $M_{2 v}$ can be compared with the predictions based on charge exchange experiments $[42,43]$. An agreement between those two evaluations will increase the knowledge of the reaction mechanisms and the nuclear structure aspects involved in $2 v \beta \beta$.

The data set considered for the $2 \nu \beta \beta$ analysis was taken between November 9, 2011, and March 21, 2012, corresponding to an exposure of $5.04 \mathrm{~kg} \cdot \mathrm{yr}$. The collected data were processed following the steps already described in Section 2 . The $2 v \beta \beta$ analysis was performed in the energy range between 600 and $1800 \mathrm{keV}$. Below $600 \mathrm{keV}$ indeed the GERDA energy spectrum is largely dominated by ${ }^{39} \mathrm{Ar}$ decay. From Monte Carlo simulations, the probability of $2 \nu \beta \beta$ decay fully contained in the active volume of the detectors and producing a total energy release between 600 and $1800 \mathrm{keV}$ is about $63.5 \%$. The probability of releasing energy in the region 


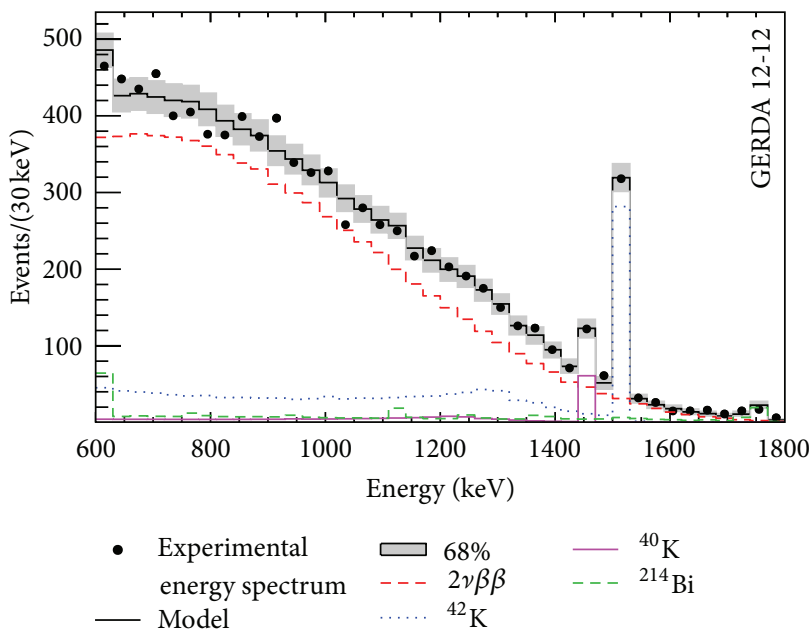

(a)

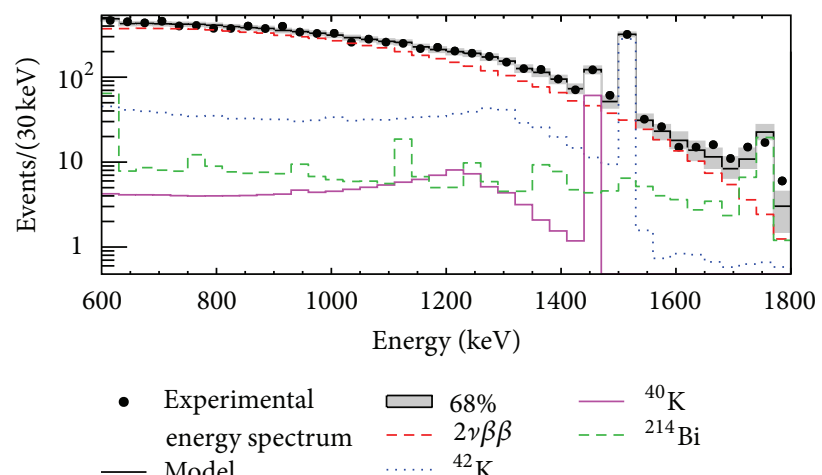

(b)

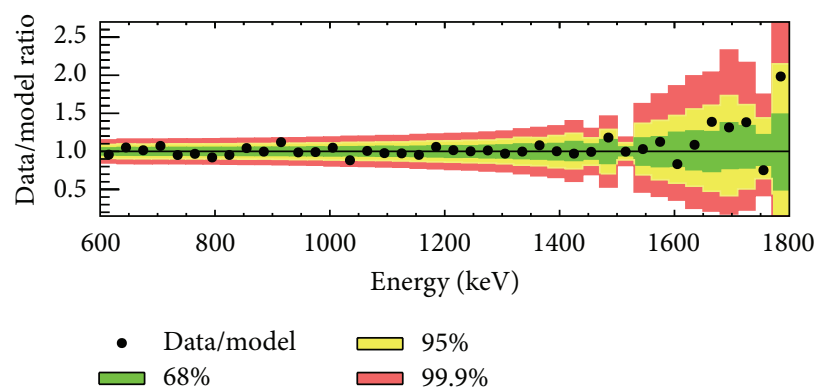

(c)

FiguRE 3: (a) shows the experimental data (black markers) and the best fit model (black histogram) for the sum of the semicoaxial detectors in linear scale and (b) in logarithmic scale. Individual components are shown with colored histograms. The shaded band covers the 68\% probability range for the model expectation. (c) gives the ratio between experimental data and the prediction of the best fit model. The smallest intervals containing the $68 \%, 95 \%$, and $99.9 \%$ probability for the expectation are shown in green, yellow, and red regions, respectively. From [31].

above $1800 \mathrm{keV}$ is less than $0.02 \%$ : therefore the energy region above $1800 \mathrm{keV}$ gives no information on the $2 \nu \beta \beta$ process. The total number of events in the selected energy window is 8796. The experimental spectra of the diodes were analyzed following a binned maximum likelihood approach described in [44]. The analysis region was divided into 40 bins, of $30 \mathrm{keV}$ each. A global model was fitted to the observed energy spectra. It includes the ${ }^{76} \mathrm{Ge} 2 \nu \beta \beta$ decay and three main background components: ${ }^{42} \mathrm{~K},{ }^{214} \mathrm{Bi}$, and ${ }^{40} \mathrm{~K}$. The presence of such background sources was established by the observation of their characteristic $\gamma$ lines in the energy spectra: $1525 \mathrm{keV}$ from ${ }^{42} \mathrm{~K} \mathrm{keV}, 1460 \mathrm{keV}$ from ${ }^{40} \mathrm{~K}$, and $609 \mathrm{keV}$ and $1764 \mathrm{keV}$ due to ${ }^{214} \mathrm{Bi}$. The $2 \nu \beta \beta$ decay half-life is common in the fit to the six semicoaxial detector spectra. The intensities of the background components are independent for each detector. The shapes of the energy spectra for the signal and the three backgrounds contamination are obtained from the Monte Carlo simulation, separately calculated for each detector. The simulation is performed using the Mage code [45] based on GEANT $4[46,47]$. The spectrum of the two electrons emitted in the ${ }^{76} \mathrm{Ge} 2 \nu \beta \beta$ decay follows the distribution of [3] as implemented in the DECAY0 [48] code. The ${ }^{42} \mathrm{~K}$ activity is uniformly distributed in the LAr volume. The actual position of the ${ }^{40} \mathrm{~K}$ and ${ }^{214} \mathrm{Bi}$ emitters is not known very well: in the Monte Carlo simulation a "close source" assumption was made. The spectral fit was performed using the Bayesian Analysis Toolkit (BAT) [49]. Well-chosen prior probability density functions (PDF) were given for $T_{1 / 2}^{2 v}$, for the active volume fractions and ${ }^{76} \mathrm{Ge}$ isotopic abundances of the germanium diodes. Figure 3 shows the data together with the best fit model (black histogram) with its 68\% uncertainties. The best fit model returns 8797.0 events in comparison to 8796 data events, with the following components: ${ }^{76} \mathrm{Ge} 2 \nu \beta \beta$ (79\%), ${ }^{42} \mathrm{~K}(14.1 \%),{ }^{218} \mathrm{Bi}(3.8 \%)$, and ${ }^{40} \mathrm{~K}(2.1 \%)$. The signalto-background ratio in the region $600-1800 \mathrm{keV}$ is around 4 to 1 , much better than that for any past experiment observing the ${ }^{76} \mathrm{Ge} 2 \nu \beta \beta$ decay. The best half-life estimate, having marginalized over all nuisance parameters, gives

$$
T_{1 / 2}^{2 v}=\left(1.84_{-0.08 \text { fit }}^{+0.09}{ }_{-0.06 \text { syst }}^{+0.11}\right) \times 10^{21} \mathrm{yr} .
$$

The first error comes from the statistics and the marginalization of the nuisance parameters, while the second relates to the systematic uncertainties. Active masses and ${ }^{76} \mathrm{Ge}$ isotopic abundances drive the uncertainties coming from the 


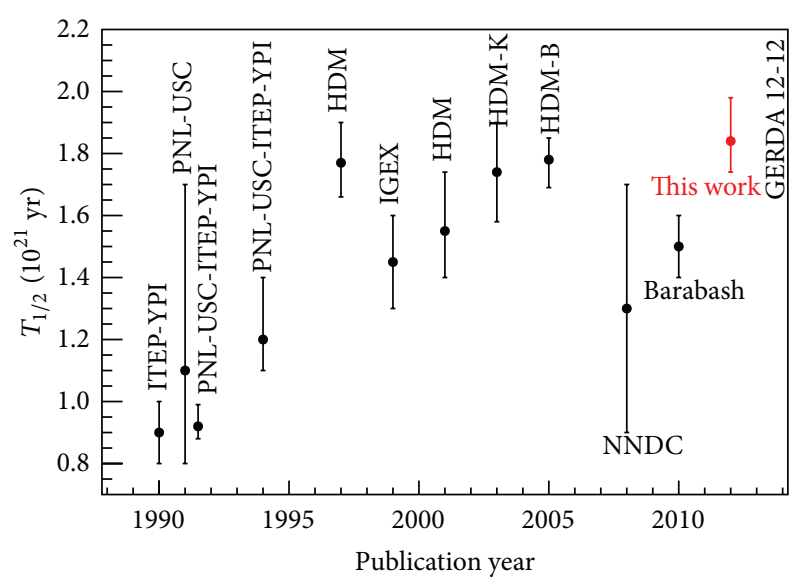

FIgURE 4: Experimental results for $T_{1 / 2}^{2 v}$ of ${ }^{76} \mathrm{Ge}$ versus publication year. The plot includes results from the experiments ITEP-YPI [55], PNL-USC [56], PNL-USC-ITEP-YPI [57, 58], Heildelberg-Moscow (HDM) $[15,59]$, and IGex $[17,18]$ as well as reanalysis of the HDM data by Dörr and Klapdor-Kleingrothaus [60] (HDM-K) and by Bakalyarov et al. [61] (HDM-B). Recommended values coming from a global analysis are also shown (NNDC [62], Barabash [2]). From [31].

fit procedure, while the dominant systematic uncertainties are due to the background model.

A compilation of the ${ }^{76} \mathrm{Ge} T_{1 / 2}^{2 v}$ results from earlier experiments plotted versus the publication year, nine experimental measurements and two weighted averages, is shown in Figure 4. A trend toward longer $T_{1 / 2}^{2 v}$ values is clearly seen. The effect is probably related to the signal-to-background ratio increase over the years. This also explains why the total GERDA uncertainty is comparable to what was obtained by HDM in spite of the much smaller exposure. From the present GERDA result and using the improved electron wave functions reported in [50], it is possible to calculate the nuclear matrix element for the ${ }^{76} \mathrm{Ge} 2 \nu \beta \beta$ decay: $M_{2 v}=0.133_{-0.005}^{+0.004} \mathrm{MeV}^{-1}$. The value is consistent with ${ }^{76} \mathrm{Ge} M_{2 v}$ extracted from the charge exchange reactions $\left(\mathrm{d},{ }^{2} \mathrm{He}\right)$ and $\left({ }^{3} \mathrm{He}, \mathrm{t}\right)[42,43]$.

3.2. Background Decomposition. The characteristic signature of the $0 \nu \beta \beta$ process is the presence of a peak at $Q_{\beta \beta}$. All other parts of the energy spectrum are to be considered as background. It is obvious that a good knowledge of the background composition is rather important in order to extract a possible signal or in case of no signal events to obtain a lower limit on $T_{1 / 2}^{0 \nu}$. Background decomposition is also useful to derive a strategy for background suppression. A comprehensive analysis of the background decomposition of the data was made using $18.50 \mathrm{~kg} \cdot \mathrm{yr}$ over a total collected exposure of $21.60 \mathrm{~kg} \cdot \mathrm{yr}$. That exposure was subdivided into three data sets: GOLD-coax with $15.40 \mathrm{~kg} \cdot \mathrm{yr}$, SILVER-coax with $1.30 \mathrm{~kg} \cdot \mathrm{yr}$, and $B E G e$ with $1.80 \mathrm{~kg} \cdot \mathrm{yr}$.

It is worth reminding that a $40 \mathrm{keV}$ energy window between $2019 \mathrm{keV}$ and $2049 \mathrm{keV}$ remained blinded up to the collection of $20 \mathrm{~kg} \cdot \mathrm{yr}$; afterwards it was partially unblinded, with the opening of a $30 \mathrm{keV}$ region for the semicoaxial detectors and a $32 \mathrm{keV}$ wide window for the BEGe detectors, respectively. The energy widths of the final blind windows (10 keV for semicoaxial detectors and $8 \mathrm{keV}$ for BEGe detectors) are dictated by the different energy resolution of the two types of detectors. The partial unblinding was done in order to test the background model predictions.

The energy spectra obtained from enriched semicoaxial, enriched BEGe, and natural abundance semicoaxial detectors are shown in Figure 5, respectively. The low energy part up to $565 \mathrm{keV}$ is dominated by cosmogenic ${ }^{39} \mathrm{Ar} \beta$-decay in all spectra, with differences in shape between semicoaxial and BEGe type detectors due to geometry and of $\mathrm{n}^{+}$dead layer thickness variations among the diodes. Between $600 \mathrm{keV}$ and $1500 \mathrm{keV}$ the enriched detectors spectra show an enhanced continuous spectrum due to $2 \nu \beta \beta$ decay. $\gamma$-lines from the decays of ${ }^{40} \mathrm{~K}$ and ${ }^{42} \mathrm{~K}$ can be identified in all spectra. The enriched detectors spectra contain also lines coming from ${ }^{208} \mathrm{Tl},{ }^{214} \mathrm{Bi}$ (visible in Figure 5), ${ }^{60} \mathrm{Co},{ }^{214} \mathrm{~Pb}$, and ${ }^{228} \mathrm{Ac}$ (not visible in Figure 5) decays. A peak-like structure around $5.3 \mathrm{MeV}$ is visible in the enriched semicoaxial diodes spectra and partially also in that of the natural one. Such feature can be attributed to the ${ }^{210}$ Po decay in the detector $\mathrm{p}^{+}$surfaces. Additional peak-like structures at energies of $4.7 \mathrm{MeV}$, $5.4 \mathrm{MeV}$, and $5.9 \mathrm{MeV}$ are identified as indicated in Figure 5. They are attributed to ${ }^{226} \mathrm{Ra},{ }^{222} \mathrm{Rn}$, and ${ }^{218} \mathrm{Po} \alpha$ decays on the detector $\mathrm{p}^{+}$surface, respectively.

Background components clearly identified in the energy spectra (see the previous discussion) or that were known to be present near the detectors from the material screening were simulated using the MAGE code. The expected BIs due to the neutron and muon fluxes at the LNGS underground laboratories have been estimated to be of the order of $10^{-5} \mathrm{cts} /(\mathrm{keV} \cdot \mathrm{kg} \cdot \mathrm{yr})$ [51] and $10^{-4} \mathrm{cts} /(\mathrm{keV} \cdot \mathrm{kg} \cdot \mathrm{yr})$ [52] and were not considered in the analysis.

The energy spectrum between $570 \mathrm{keV}$ and $1500 \mathrm{keV}$ can be explained in terms of the same background components described in the Section 3.1. The events in the $3.5 \mathrm{MeV}$ to $7.5 \mathrm{MeV}$ energy region are expected to come from $\alpha$ emitting isotopes in the ${ }^{226} \mathrm{Ra}$ decay chain, which can be broken at ${ }^{210} \mathrm{~Pb}$ and at ${ }^{210} \mathrm{Po}$ due to their long half-lives of $22.3 \mathrm{yr}$ and 138.4 days, respectively. An analysis of the time distribution of the collected events demonstrates the presence of the ${ }^{210}$ Po isotope. The event rate distribution is well described by an exponentially decreasing plus a constant rate model: the half-life is $138.4 \pm 0.4$ days, in nice agreement with the literature value for the half-life of ${ }^{210}$ Po. In Figure 6 the energy spectrum between $3.5 \mathrm{MeV}$ and $7.5 \mathrm{MeV}$ is shown (black markers) together with the best fit model (black histogram). The spectrum is well described assuming the presence of ${ }^{210}$ Po on the surface of the diodes and also of ${ }^{226} \mathrm{Ra}$ and its short lived daughter nuclei $\left({ }^{222} \mathrm{Rn},{ }^{218} \mathrm{Po}\right)$ on the $\mathrm{p}^{+}$surface and in LAr. In order to describe the data, different layer thicknesses of the $\mathrm{p}^{+}$surface were taken into account (from 300 to $600 \mathrm{~nm}$ ). In the other panel the ratio of data and model are shown; the colored bands give the smallest interval probability for the model expectation.

The best fit model and the single background components together with the observed data spectrum from 


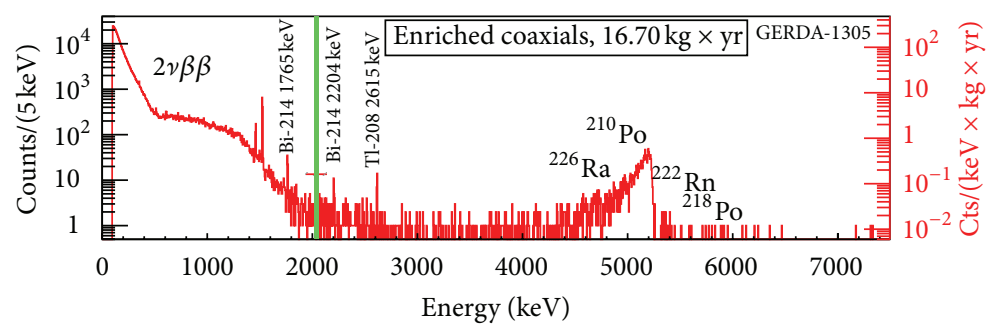

(a)

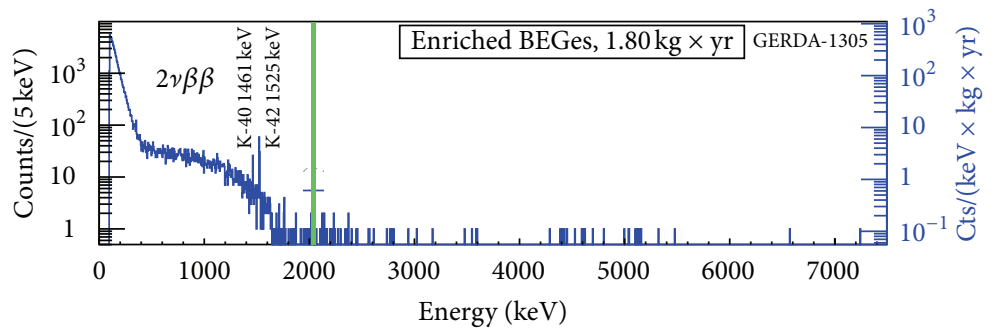

(b)

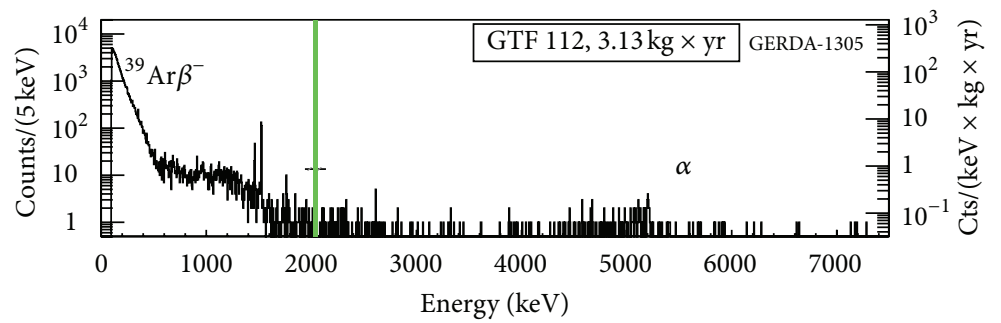

(c)

Figure 5: Spectra obtained using all the enriched semicoaxial (a), BEGe (b), and nonenriched (c) detectors. The blinding window of 40 keV around the $Q_{\beta \beta}$ is indicated with the green band. The bars in the color of the histogram around the green band represent the $200 \mathrm{keV}$ region from which the BI of the dataset is computed. From [28].

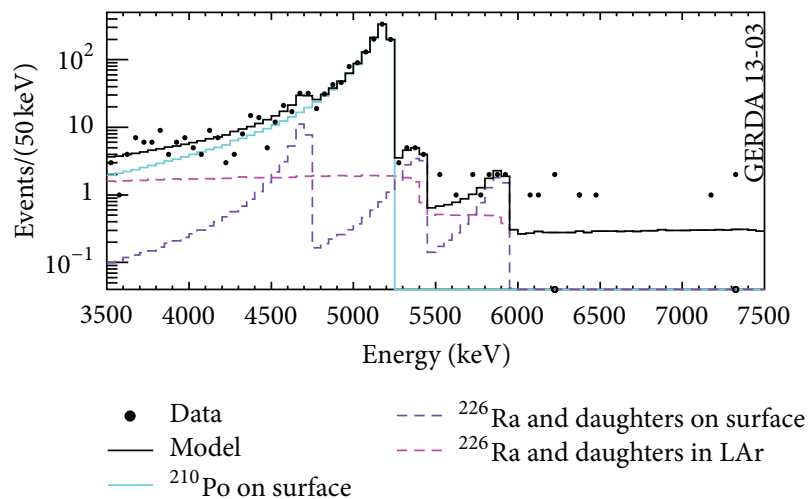

(a)

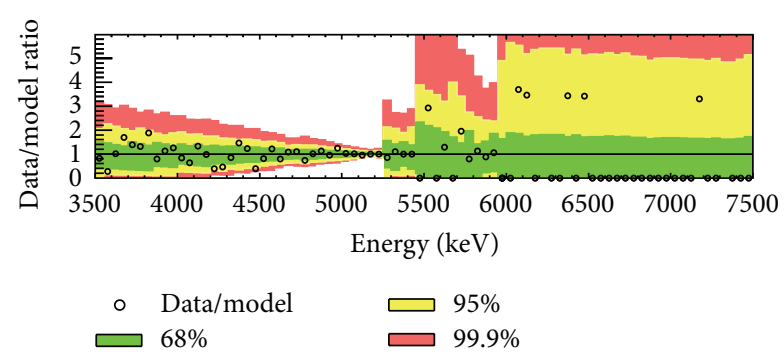

凹 $68 \%$ $99.9 \%$

FIGURE 6: (a) shows the best fit model (black histogram) and the observed spectrum (black dots) for the GOLD-coax data set. The other curves represent the individual components of the model. (b) shows the data over model ratio (empty points) and the smallest intervals containing 68\% (green), 95\% (yellow), and 99.9\% (red) probability for the model expectation. From [28].

$570 \mathrm{keV}$ to $1650 \mathrm{keV}$ and from $1650 \mathrm{keV}$ to $3750 \mathrm{keV}$ is shown in Figure 7. The best fit model around the $Q_{\beta \beta}$ for the GOLD-coax data is shown in Figure 8. The spectral shape of the model is flat around $Q_{\beta \beta}$, and no peaks are predicted in the blinded regions. The predicted BI around
$Q_{\beta \beta}$ is $1.85_{-0.09}^{+0.08} \times 10^{-2} \mathrm{cts} /(\mathrm{keV} \cdot \mathrm{kg} \cdot \mathrm{yr})$. The main background contributions come from ${ }^{214} \mathrm{Bi}$ and ${ }^{228} \mathrm{Th}$ in the detector assembly, ${ }^{42} \mathrm{~K}$ homogeneously distributed in LAr and finally from $\alpha$ emitters. The predicted BI nicely agrees with the same quantity derived from the data interpolation in the energy 


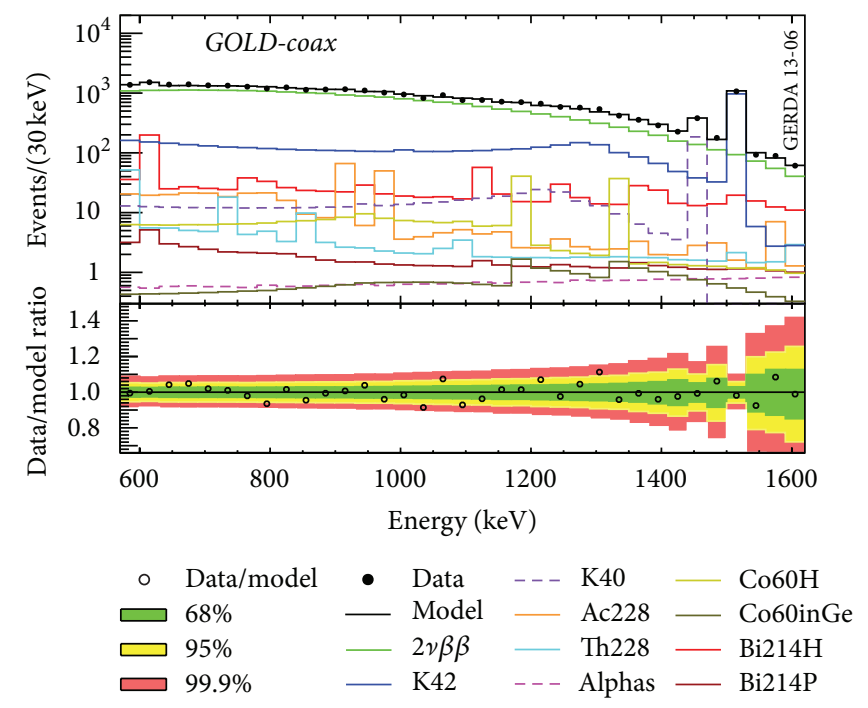

(a)

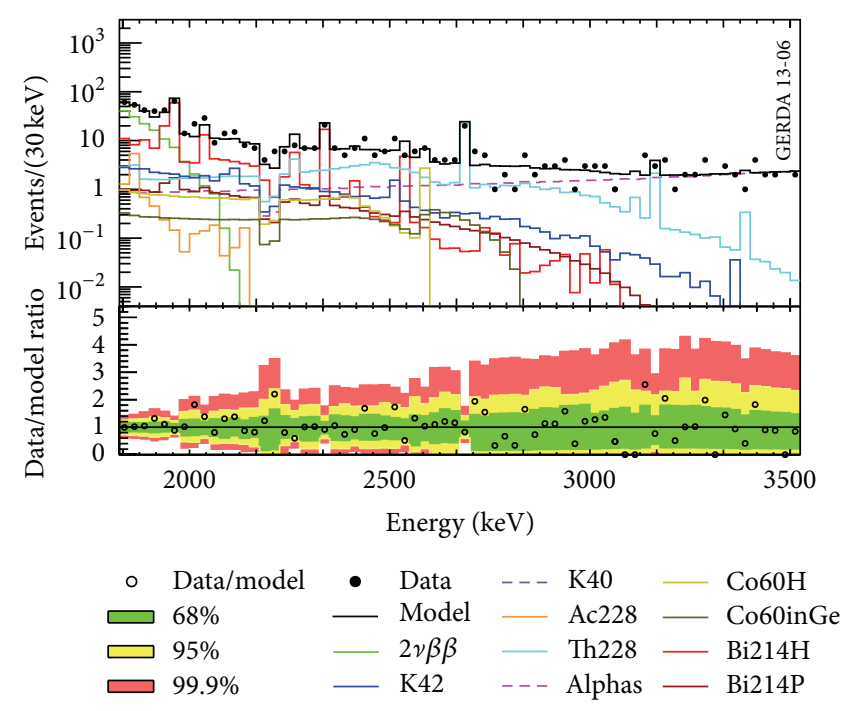

(b)

Figure 7: (a) shows the background decomposition (black histogram) and the observed spectrum (black dots) using the best fit minimum model for the GOLD-coax data set in the energy range from $570 \mathrm{keV}$ to $1650 \mathrm{keV}$. The lower panel shows the data over model ratio (empty points) and the smallest intervals containing 68\% (green), 95\% (yellow), and 99.9\% (red) probability for the model expectation. (b) shows the same for the energy range from $1650 \mathrm{keV}$ to $3750 \mathrm{keV}$. From [28].

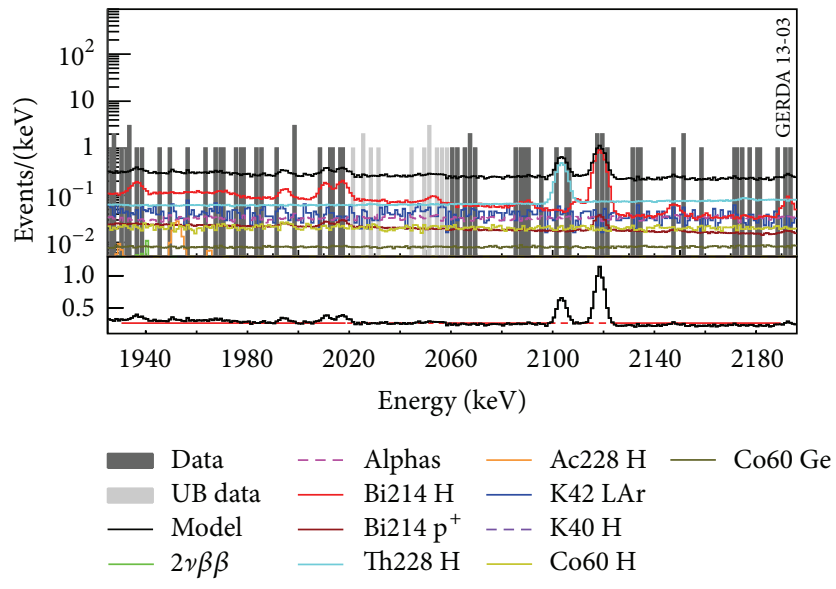

(a)

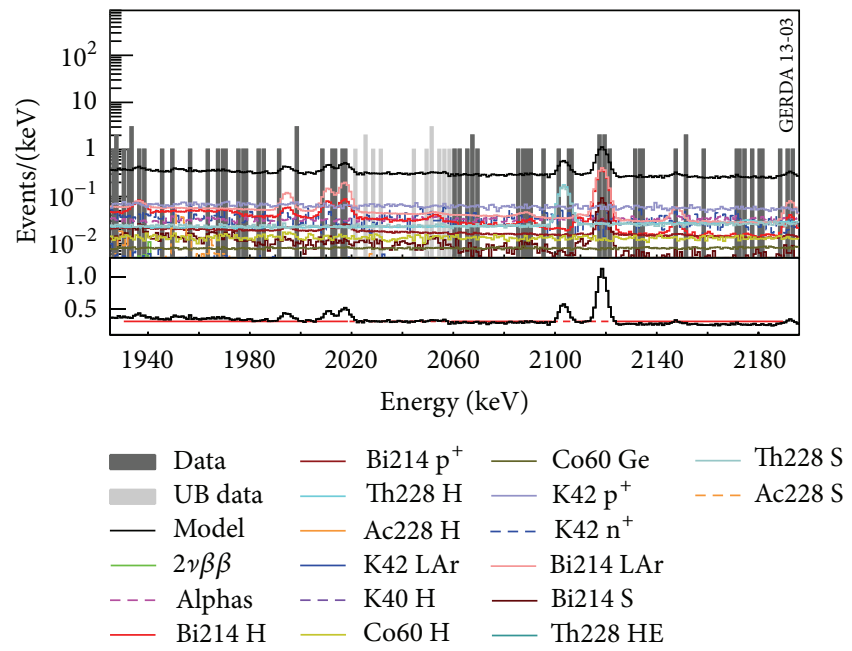

(b)

FIGURE 8: Experimental spectrum with minimum (a) model around $Q_{\beta \beta}$ for the GOLD-coax data set. (a) shows the individual contributions of the considered background sources (colored curves) and the model prediction (black curve). The events colored in gray were used in the analysis, while the light gray shaded (unblinded data, UB data) ones have not been used in the analysis. The lower panel shows the best fit model fitted with a constant. In the fit, the peak areas predicted by the model and the $40 \mathrm{keV}$ blinding window are not considered. (b) shows the same experimental spectrum compared with maximum model around $Q_{\beta \beta}$ for the GOLD-coax data set. From [28].

intervals 1930-2019 keV, 2059-2099 keV, 2109-2114 keV, and $2124-2190 \mathrm{keV}$ for a total width of $200 \mathrm{keV}: 1.75_{-0.24}^{+0.26} \times$ $10^{-2} \mathrm{cts} /(\mathrm{keV} \cdot \mathrm{kg} \cdot \mathrm{yr})$. This BI evaluation window excludes the central $40 \mathrm{keV}$ window around $Q_{\beta \beta}$ and the regions within $\pm 5 \mathrm{keV}$ of the single escape peak from ${ }^{208} \mathrm{Tl}$ at $2104 \mathrm{keV}$ and the ${ }^{214} \mathrm{Bi} \gamma$ line at $2119 \mathrm{keV}$. The model discussed here gives a satisfactory description of the spectrum without any medium distance and distant contaminations. It is called minimum model. It is also possible to build a model with a fair description of the data leaving the possibility to have contamination sources at medium and long distances. The main difference between the two models is the number of events deposited on the $\mathrm{p}^{+}$surface of the detectors. This last model is called maximum model (see Figure $8(\mathrm{~b})$ ). The predicted BI of the maximum model is $2.19_{-0.12}^{+0.19} \times 10^{-2} \mathrm{cts} /(\mathrm{keV} \cdot \mathrm{kg} \cdot \mathrm{yr})$, not very far from the minimum model estimation. To conclude 


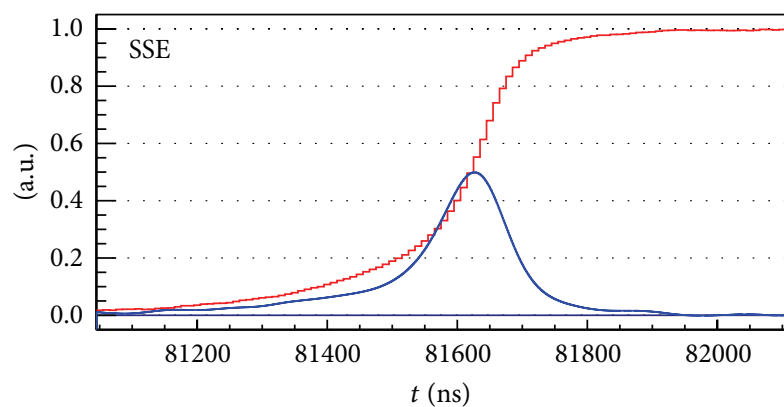

(a)

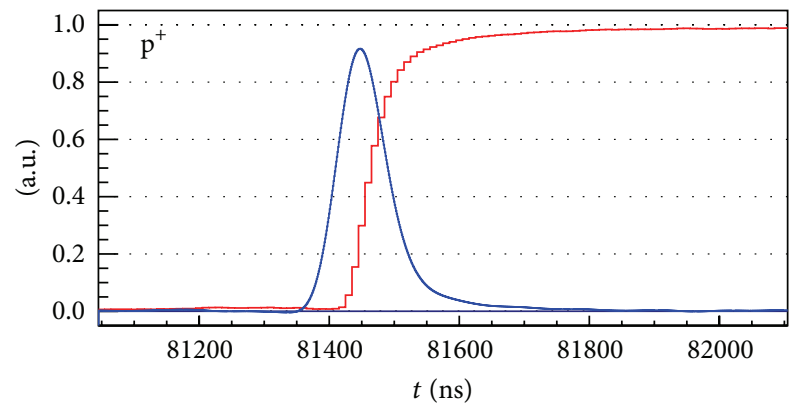

- Charge

(c)

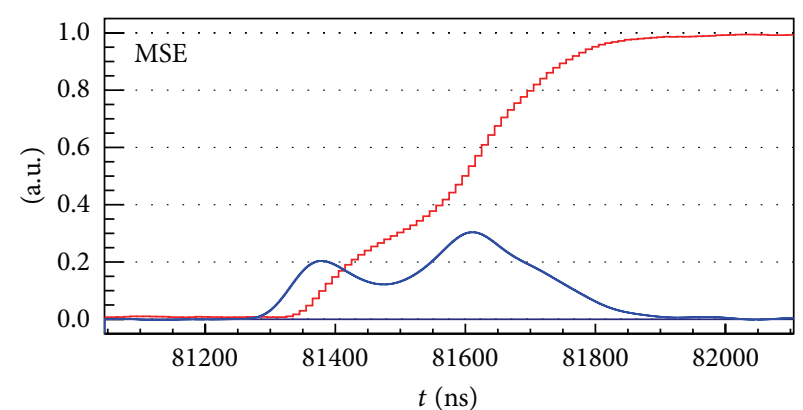

(b)

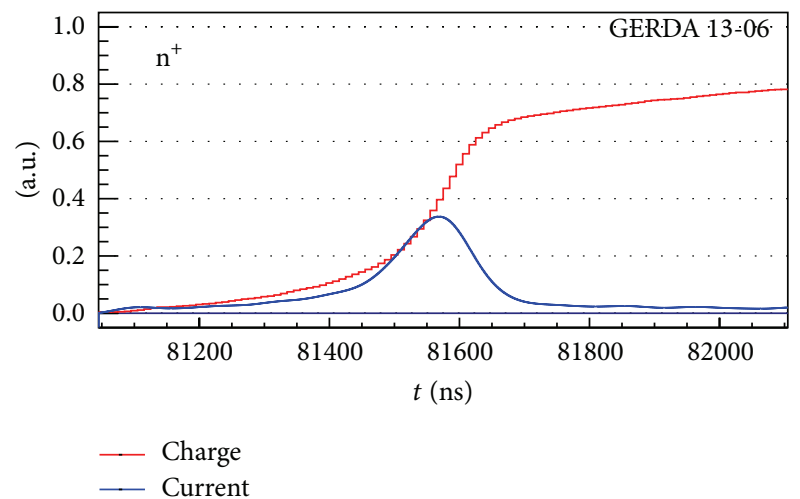

(d)

FiguRE 9: Examples of pulse traces taken from SSE data (a), MSE (b), $\mathrm{p}^{+}$electrode event (c), and $\mathrm{n}^{+}$surface event (d). The pulse traces are normalized in order to have the maximum of the charge pulse equal to one, current pulses have equal integrals. From [32].

we have to say that all contaminations expected from material screening are seen in the background spectra and are taken into account in the models.

As mentioned, the $40 \mathrm{keV}$ blinded region was partially unblinded leaving a central $10 \mathrm{keV}$ for semicoaxial detectors (8 keV for BEGe detectors) window still blind. In total 13 events were observed in the unblinded $30 \mathrm{keV}$ window of the GOLD-coax data set. The predictions in this window were 8.6 events for the first model and 10.3 for the second one. The agreement between predictions and observed number of events in the unblinded region is rather good.

3.3. Pulse Shape Discrimination Analysis. Signal events have a different topology with respect to a large part of the background events. Due to the limited range of the electrons in germanium (a few $\mathrm{mm}$ ), the two $0 \nu \beta \beta$ electrons release their energy in a well-localized space area inside the detectors. This type of events is SSE. The background in the region of interest is largely due to $\gamma$ s. The latter often interact more than once in the detector at points separated by several centimeters. This type of events is MSE. This different topology is usually registered in the rising part of the recorded signal and the distinction can be facilitated by the particular electric field inside the detector. The procedure of selecting SSE from MSE is called PSD analysis. In GERDA, two different PSD methods were used: one optimized for the BEGe detectors and the other for the semicoaxial diodes.

In the case of the BEGe we consider two measured quantities: the energy $E$ of the event (proportional to the maximum of the charge pulse) and then the maximum $(A)$ of the current pulse. The current is measured differentiating the charge pulse. Then the ratio $A / E$ is built and used as a PSD variable $[53,54]$.

The physical justification of such a variable is easy to understand looking to Figure 2. The weighting potential sharply rises in the small region around the $\mathrm{p}^{+}$contact; for that reason, in a single interaction, mainly holes contribute to the induced signal on the readout electrode. Single interactions in different places of the detectors produce charge pulses which differ only from the collection time and the total released energy. If the energy release happens in different places of the BEGe (as for a $\gamma$ interaction) the output pulse will be the convolution of all the different energy deposition and contain this information in the shape of its rising edge.

An application of this simple idea is shown in Figure 9. In Figure 9(a) a charge pulse trace for a SSE event is shown with its current pulse superimposed. For a SSE in the bulk detector an almost Gaussian distribution of $A / E$ is expected with a width dominated by the noise of the readout electronics. The situation for a MSE event is depicted in Figure 9(b): for the same total energy $E$, the maximum current amplitude will be smaller due to the various energy depositions in different places of the detector and at different times. Examples of $\mathrm{p}^{+}$ electrode and $\mathrm{n}^{+}$surface events are shown in the bottom right and left plots of Figure 9, respectively.

The $A / E$ method performance was determined using calibration data. A ${ }^{228} \mathrm{Th}$ calibration spectrum contains a line at $2614.5 \mathrm{keV}$ from ${ }^{214} \mathrm{Tl}$ decays, a double escape peak 


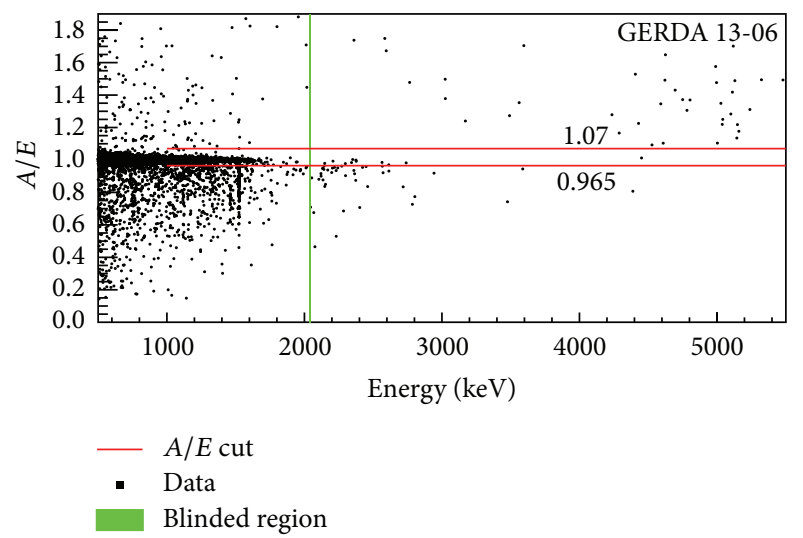

Figure 10: $A / E$ versus energy for the BEGe data set. The accepted region is that inside the two red lines (low cut at $A / E=0.965$, high cut at $A / E=1.07)$. The blinded region is given by the green band. From [32].

(DEP) at $1592.5 \mathrm{keV}$ dominated by SSE, while the full energy peak (FEP) at $1620.7 \mathrm{keV}$ or the single escape peak (SEP) at $2103.5 \mathrm{keV}$ are dominated by MSE. (If a $\gamma$ ray has sufficiently high energy (several MeV), the results of pair production are evident in the electron spectrum. If the two annihilation $\gamma \mathrm{s}$ leave the detector, only the electron and positron energies are deposited. The net effect is to add a peak to the spectrum, called double escape peak, located at an energy of $\sim 1.02 \mathrm{MeV}$ below the full energy peak. The full energy peak corresponds to the total energy of the primary $\gamma$. If instead only one annihilation $\gamma$ escapes from the detector another peak is added, called single energy peak, which now appears at an energy of $\sim 0.511 \mathrm{MeV}$ below the full energy peak.) Further checks come from the use of the $2 \nu \beta \beta$ events which are genuine SSE distributed homogeneously in the detector. If one determines the mean value of the $A / E$ distribution using DEP from calibration data, then it is possible to rescale the $A / E$ of any event by that mean value. In this manner, the $A / E$ distribution of all SSE (whatever is their origin) will be centered at 1 ; MSE and $\mathrm{n}^{+}$surface events will populate the region below 1 while $\mathrm{p}^{+}$electrode events the region above 1.

An $A / E$ distribution plotted versus energy is shown in Figure 10. The red lines correspond to $A / E<0.965$ ("low $A / E$ cut") and $A / E>1.07$ ("high $A / E$ cut"), respectively. The low $A / E$ cut has the aim to reject MSE and $\mathrm{n}^{+}$events; its value is chosen to minimize at values below $1 \%$ the efficiency loss of the $A / E$ Gaussian function for energy above $1 \mathrm{MeV}$. The high $A / E$ cut rejects $\mathrm{p}^{+}$events; its value is twice larger than the low cut due to the much lower number and better separation of $\mathrm{p}^{+}$electrode events.

With the aforementioned cut values, the efficiency of the $A / E$ method for $0 \nu \beta \beta$ events is $0.92 \pm 0.02$ as given by DEP events from calibration data and cross-checked by the use of $2 v \beta \beta$ events. The $A / E$ method rejects about $80 \%$ of the background events at $Q_{\beta \beta}$. Figure 11 shows the BEGe data energy spectrum before and after the PSD cuts. With an exposure of $2.4 \mathrm{~kg} \cdot \mathrm{yr}$, seven out of 40 events in the $400 \mathrm{keV}$ wide region around $Q_{\beta \beta}$ are kept. The $\mathrm{BI}$ for BEGe

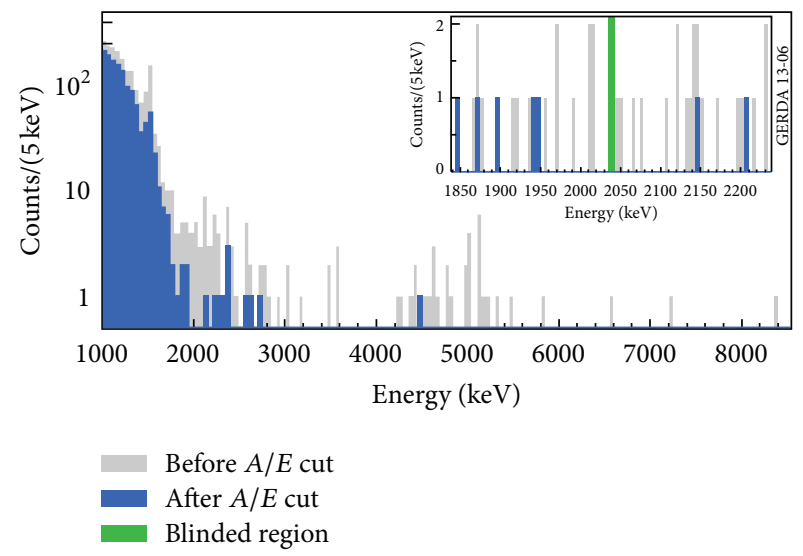

FIGURE 11: Energy spectrum of the BEGe data set. The gray histogram shows the data before PSD cut and the blue one after PSD cut. The inset shows the region $Q_{\beta \beta}$, with the $8 \mathrm{keV}$ blinded region in green. From [32].

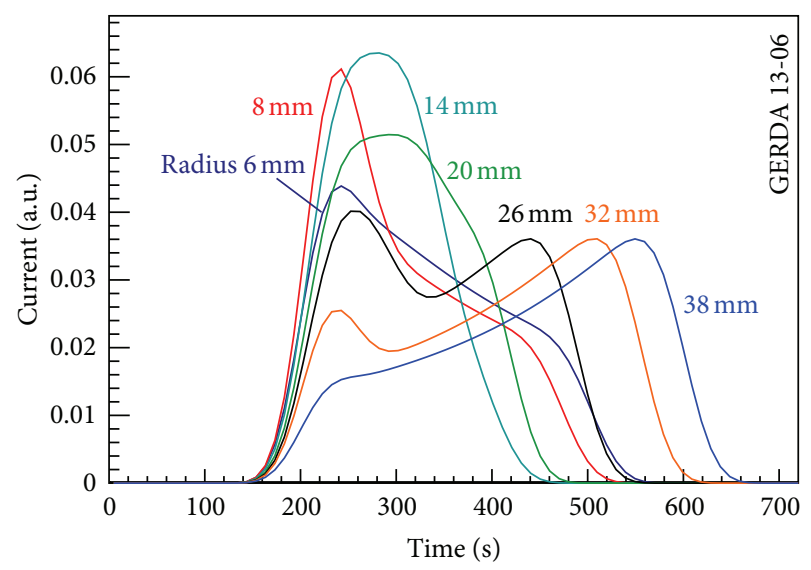

FIGURE 12: Simulated current pulse shapes for SSE in a semicoaxial detector taken at various places inside the diode. The places vary from the outer $\mathrm{n}^{+}$surface towards the bore hole $\left(\mathrm{n}^{+}\right.$surface) along a radial line at the midplane of the detector. From [32].

detectors in this interval is reduced from $(0.042 \pm 0.007)$ to $\left(0.007_{-0.002}^{+0.004}\right) \mathrm{cts} /(\mathrm{keV} \cdot \mathrm{kg} \cdot \mathrm{yr})$.

For semicoaxial detectors the situation is more complicated. The weighting potential (see Figure 2(a)) also peaks at the $\mathrm{p}^{+}$contact but the gradient is lower and hence a larger part of the volume, with respect to the BEGe, is important for the charge pulse. For an interaction which happens in the bulk of the detector, both electrons and holes contribute. All these complications make the semicoaxial less good in PSD with respect to the BEGe. Simulated current pulse shapes for SSE taken in various positions starting from the outer $\mathrm{n}^{+}$surface and going towards the bore hole $\left(\mathrm{n}^{+}\right.$surface) along a radial line at the midplane of the detector are shown in Figure 12. The pulse shape behaviour is rather complicated so a simple $A / E$ variable cannot efficiently discriminate between SSE and MSE.

For the semicoaxial detectors a PSD analysis based on neural network was applied. The selected algorithm called 


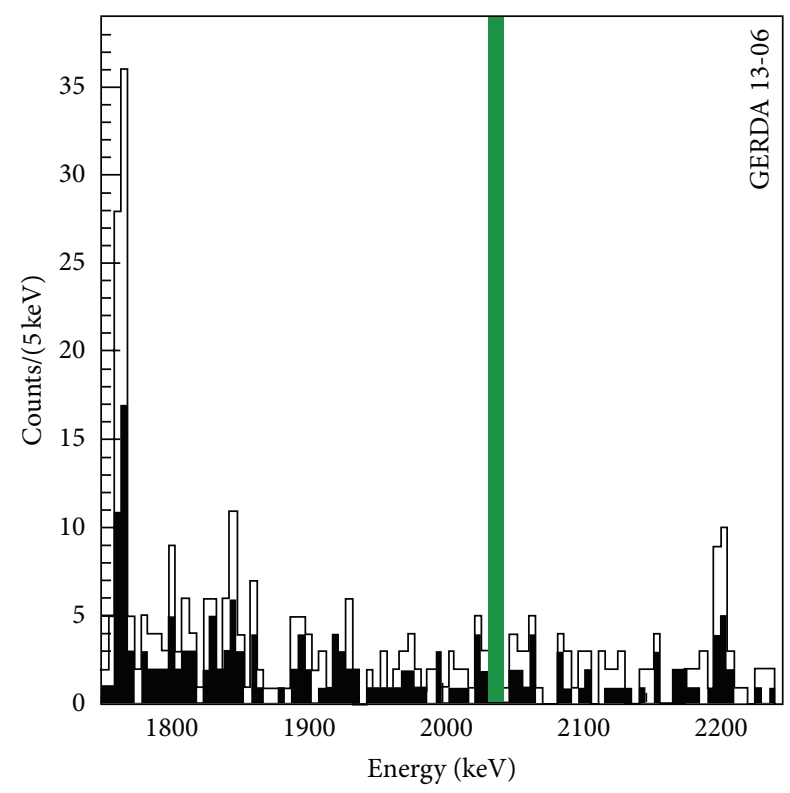

$\boxminus$ Without PSD
With PSD

FIGURE 13: Energy spectrum of semicoaxial detectors without (empty histogram) and with (black histogram) neural network PSD selection. From [32].

TMlpANN (present inside the TMVA [63] toolkit implemented in Rоoт) is based on multilayer perceptron. Two hidden layers, with 50 neurons each, are used.

The rising part of the charge pulse is used for this neural network analysis. The input parameters are the times when the pulses reach $1,3,5, \ldots, 99 \%$ height. The time, at which $50 \%$ of the charge of the pulse have been seen, serves as a reference. Due to the $100 \mathrm{MHz}$ sampling frequency, a linear interpolation is required between two consecutive time bins in order to determine the corresponding time points. For the training calibration data are used. The output of the neural network is a number between 0 for background and 1 for signal like events. The cut values are chosen in order to have detector per detector a DEP survival probability fixed at $90 \%$. All possible deviations from $90 \%$ were taken into account (energy dependence, volume effect due to the different distribution of DEP and $0 \nu \beta \beta$ events, etc.) and combined quadratically. The final result for the $0 \nu \beta \beta$ efficiency of the neural network is $0.90_{-0.09}^{+0.05}$. About $45 \%$ of the events in a $230 \mathrm{keV}$ window around $Q_{\beta \beta}$ are classified as background and rejected.

The energy spectrum collected by the semicoaxial detectors before and after the neural network PSD selection is shown in Figure 13.

3.4. Limit on the Half-Life of the $0 \nu \beta \beta$ Decay Process in ${ }^{76} \mathrm{Ge}$. The total exposure useful for the $0 \nu \beta \beta$ analysis was of $21.60 \mathrm{~kg} \cdot \mathrm{yr}$ of ${ }^{\mathrm{enr}} \mathrm{Ge}$ detectors collected from November 9, 2011, to May 21, 2013 (492.3 live days). The collected data were processed following the steps described in Section 2. The deposited energy is reconstructed by a digital filter with
semi-Gaussian shaping. Events generated by discharges or due to electromagnetic noise are rejected by a set of quality cuts. The analysis cuts applied to the data were the following.

(i) Only events with an energy deposition in a single detector were accepted (anticoincidence cut). Around $Q_{\beta \beta} 15 \%$ of the background events are rejected, with no loss for $0 \nu \beta \beta$ events.

(ii) Events in the germanium detectors in coincidence within $8 \mu$ s with a signal from the muon veto are rejected (muon veto cut). This leads to a further rejection of $\sim 7 \%$ of events.

(iii) Events which are preceded or followed by another event in the same detector within less than $1 \mathrm{~ms}$ are excluded. In this manner possible events coming from the decay chain ${ }^{214} \mathrm{Bi}^{214} \mathrm{Po}$ are rejected (anti- "BiPo" cut). Less than $1 \%$ of the events around the $Q_{\beta \beta}$ are affected by this cut.

(iv) To the remaining events, the PSD cut is applied as explained in the Section 3.3.

As mentioned in Section 2.3, there was a blinded region around the $Q_{\beta \beta}$ in order to prevent from possible analysis bias. This region was open and analyzed only after the background model and all the analysis cuts (especially PSD) were frozen.

Figure 14 shows the energy spectrum around the region of interest with and without PSD selection. No excess of events were observed over a flat background distribution. Seven events were seen in the range $Q_{\beta \beta} \pm 5 \mathrm{keV}$ before the PSD cuts, while 5.1 were expected. Only 3 events survived (classified as SSE) after PSD cuts and no event remained in $Q_{\beta \beta} \pm \sigma_{E}$. To derive the number of signal counts $N^{0 v}$ a profile likelihood fit of the spectrum was performed. The fit function was given by the sum of a constant term for the background and of a Gaussian for the signal events. The profile likelihood ratio was limited to the region $T_{1 / 2}^{0 v}>0$. The best fit was obtained for $N^{0 v}=0$, that is, no excess of signal events above background. The lower limit on the half-life is

$$
T_{1 / 2}^{0 \nu}>2.1 \times 10^{25} \mathrm{yr} @ 90 \% \text { C.L. }
$$

including systematic uncertainties. This limit corresponds to $N^{0 \nu}<3.47$ events. The median sensitivity was $2.4 \times 10^{25} \mathrm{yr}$, taking into account the number of events after PSD, the efficiency, and the exposure. A Bayesian calculation using a flat prior distribution for $1 / T_{1 / 2}^{0 \nu}$ from 0 to $10^{-24} / \mathrm{yr}$ gives $T_{1 / 2}^{0 \nu}>$ $1.9 \times 10^{25}$ yr @ 90\%C.I.

As discussed before, the data collected by GERDA do not show any peak at $Q_{\beta \beta}$ and do not support the claim described in [19]. Taking the $T_{1 / 2}^{0 v}$ from [19], 5.8 signal events are expected to be observed in GERDA in an energy interval of $\pm \sigma_{E}$ with 2.0 background events after the PSD cut. In the same energy range, no events are observed. The model $\left(H_{1}\right)$ with the value of $T_{1 / 2}^{0 \nu}$ and its associated error as given from [19] gives a worse fit than the background only model $\left(H_{0}\right)$ : the Bayes factor, that is, the ratio of the posterior probabilities of the two models, is $P\left(H_{1}\right) / P\left(H_{0}\right)=0.024$. 


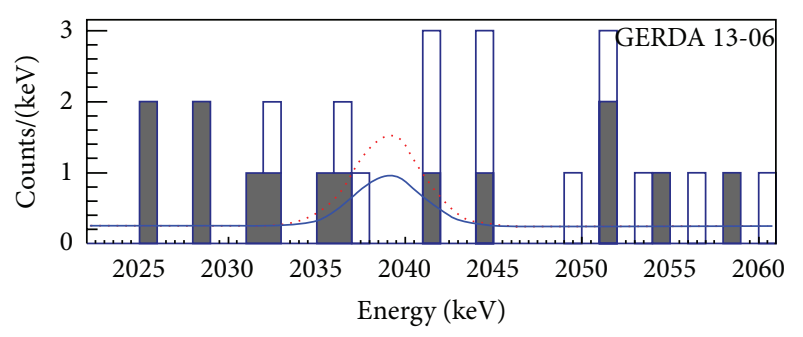

(a)

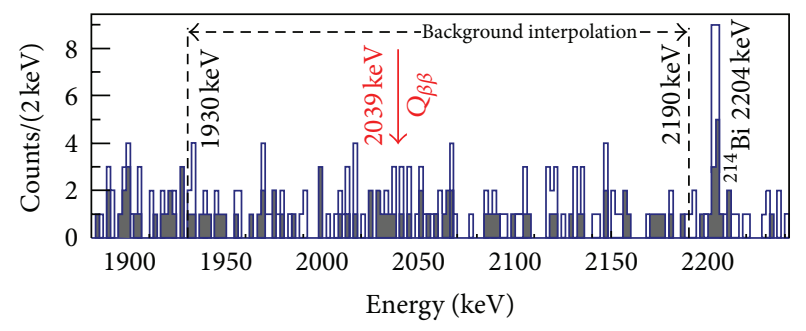

(b)

FIGURE 14: (a): Energy spectrum for the sum of all ${ }^{\mathrm{enr}}$ Ge detectors in the range $2020-2060 \mathrm{keV}$, without PSD (empty histogram) and with PSD (black histogram). The red dashed curve is the expectation based on the central value of [19]; the blue curve is instead based on the 90\% upper limit derived by GeRDA, $T_{1 / 2}^{0 \nu}=2.1 \times 10^{25} \mathrm{yr}$. (b): Energy spectrum in the range $1880-2240 \mathrm{keV}$; the vertical dashed lines indicate the interval 1930-2190 keV used for the background estimation. From [33].

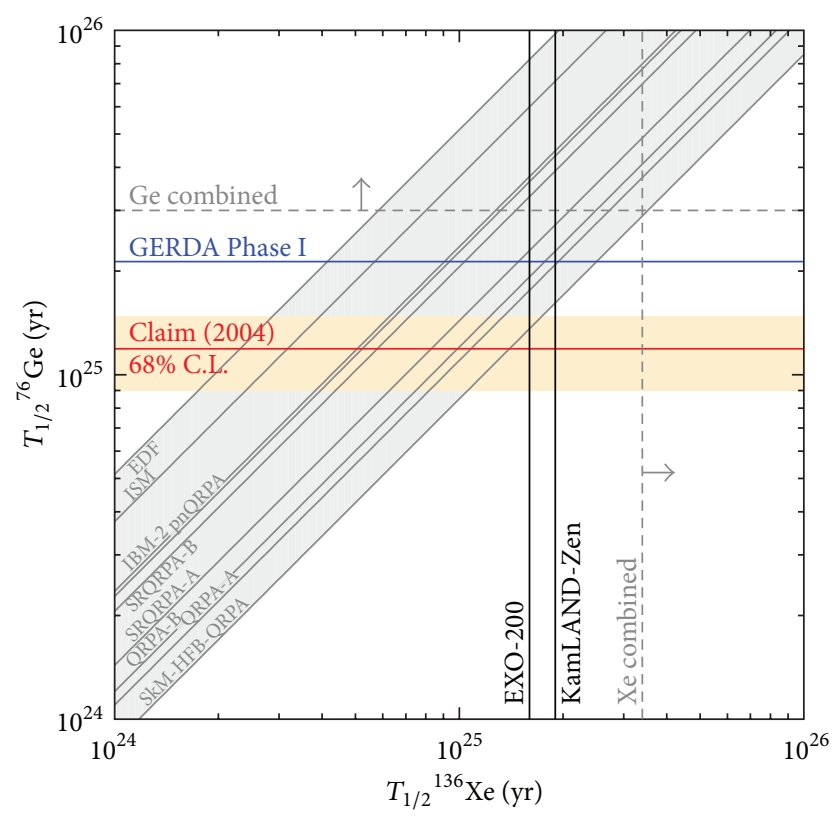

Figure 15: Limits at 90\% C.L. on $T_{1 / 2}^{0 \gamma}$ for ${ }^{76} \mathrm{Ge}$ (GERDA Phase I alone and Gerda Phase I, IGEX and HDM combined together [33]) and for ${ }^{136} \mathrm{Xe}[21,22]$. The signal claim for ${ }^{76} \mathrm{Ge}$ from [19] is also shown. The lines in the shaded gray band are the predictions for the correlation between the half-lives obtained in ${ }^{136} \mathrm{Xe}$ and in ${ }^{76} \mathrm{Ge}$ using a selection of different nuclear matrix element calculations taken from [64]. From [33].

The GERDA result is consistent with the negative results from IGEX and HDM. A combined profile likelihood fit including all these three negative results gives as best fit $N^{0 v}=$ 0 and a $90 \%$ probability limit:

$$
T_{1 / 2}^{0 v}>3.0 \times 10^{25} \mathrm{yr} @ 90 \% \text { C.L. }
$$

A Bayesian analysis gives the same limit with a Bayes factor of $2 \times 10^{-4}$ strongly disfavoring the claim. Figure 15 shows the limits set on ${ }^{76} \mathrm{Ge} T_{1 / 2}^{0 \nu}$ as determined by GERDA alone and combining GERDA with HDM and IGEx and on ${ }^{136} \mathrm{Xe} T_{1 / 2}^{0 \nu}$ as obtained by KamLAND-Zen and EXO-200 separately and combined. While the GERDA result can be compared directly with the claim of [19], in order to compare the results obtained on ${ }^{136} \mathrm{Xe}$ with the claimed signal, the assumption that the main underlying mechanism mediating $0 \nu \beta \beta$ is the exchange of light Majorana neutrinos has to be made. In this case the ratio of the half-lives of the $0 \nu \beta \beta$ decay in ${ }^{136} \mathrm{Xe}$ and ${ }^{76} \mathrm{Ge}$ is proportional to the square of the nuclear matrix ratio $M_{0 v}\left({ }^{76} \mathrm{Ge}\right) / M_{0 v}\left({ }^{136} \mathrm{Xe}\right)$, which has to be derived by theoretical calculations. The lines in the shaded band of Figure 15 are the predictions from the nuclear matrix calculations reported in [64]. The spread of the predictions on the nuclear matrix elements comes from various different approximations. It is possible, through the help of a chosen set of nuclear matrix, to make a combination of all null results from ${ }^{76} \mathrm{Ge}$ and ${ }^{136} \mathrm{Xe}$ experiments. Taking, to be conservative, the nuclear matrix element model which gives the lowest $M_{0 v}\left({ }^{136} \mathrm{Xe}\right) / M_{0 \nu}\left({ }^{76} \mathrm{Ge}\right)$ ratio, a Bayesian analysis gives a Bayes factor of 0.40 for KamLAND-Zen and 0.23 for EXO-200, to be compared to the GERDA alone result of 0.024. Putting all these experiment together, the Bayes factor becomes 0.0022 . It is important to note that other theoretical calculations might lead to even smaller ratios and so weaker exclusions.

The result of (3) can be translated into the following effective electron neutrino mass $m_{\beta \beta}<0.2-0.4 \mathrm{eV}$ having used the phase space factors from [50] and the nuclear matrix elements calculations cited in [64].

\section{Outlook}

As already explained in Section 1, the life of the GERDA experiment has been organized in two phases. Phase I was completed in the middle of May 2013 with the results discussed in the previous sections. Phase II has the goal to improve the sensitivity by an order of magnitude, that is, to reach $T_{1 / 2}^{0 \nu}>1.5 \times 10^{26}$ yr. For pure Majorana exchange, this higher sensitivity will constrain the effective electron neutrino mass to less than $100 \mathrm{meV}$; this value depending on the choice of the nuclear matrix elements. This improvement of a factor ten of the sensitivity will be obtained with the following strategy:

(i) a reduction of a factor ten of the $\mathrm{BI}$; that is, in Phase II the goal is to reach a BI of $1.0 \times 10^{-3} \mathrm{cts} /(\mathrm{keV} \cdot \mathrm{kg} \cdot \mathrm{yr})$;

(ii) adding 30 new enriched BEGe detectors $(20.1 \mathrm{~kg})$;

(iii) collecting an exposure of $100 \mathrm{~kg} \cdot \mathrm{yr}$. 
30 new enriched BEGe detectors have been already produced by Canberra Olen and fully tested by the GERDA collaboration. In order to reach the aforementioned, very low $\mathrm{BI}$ various actions will be performed. Detector holders, FE electronics cards, cables, and all the foreseen material near the detectors will be accurately selected in order to satisfy the limits imposed by the BI. Active suppression methods such as PSD analysis and the readout of the scintillation light of the liquid argon will be used to further reject the background. The work for the modification of the GERDA detector will start in the fall of 2013 and it is foreseen to be completed early in 2014, after that a commissioning phase will start and the data taking could follow in the fall of 2014.

\section{Conclusions}

In the middle of 2013, the GERDA experiment has completed its Phase I data taking. After having collected an exposure of $21.6 \mathrm{~kg} \cdot \mathrm{yr}$, no excess of $0 \nu \beta \beta$ signal events around the $Q_{\beta \beta}$ has been observed above a flat background. A lower limit at $90 \%$ C.L. has been determined for the half-life of ${ }^{76} \mathrm{Ge}$ : $T_{1 / 2}^{0 \nu}>2.1 \times 10^{25} \mathrm{yr}$. This result strongly disfavored the longstanding claim for a $0 \nu \beta \beta$ signal in ${ }^{76} \mathrm{Ge}$ with a half-life of $T_{1 / 2}^{0 \nu}=1.19 \times 10^{25} \mathrm{yr}[19]$. The Gerda experiment is now undergoing a major upgrade which will permit to increase the sensitivity by a factor of about $10\left(T_{1 / 2}^{0 v}>1.5 \times 10^{26} \mathrm{yr}\right)$ and give the possibility to explore further regions of the degenerate Majorana neutrino mass scale.

\section{References}

[1] J. Beringer, J.-F. Arguin, R. M. Barnett et al., "Review of particle physics," Physical Review D, vol. 86, no. 1, p. 1528, 2012.

[2] A. S. Barabash, "Precise half-life values for two-neutrino double- $\beta$ decay," Physical Review C, vol. 81, Article ID 035501, 7 pages, 2010.

[3] V. I. Tretyak and Y. G. Zdesenko, "Tables of double beta decay data," Atomic Data and Nuclear Data Tables, vol. 61, no. 1, pp. 43-90, 1995.

[4] V. I. Tretyak and Y. G. Zdesenko, "Tables of double beta decay data-an update," Atomic Data and Nuclear Data Tables, vol. 80, no. 1, pp. 83-116, 2002.

[5] N. Ackerman, B. Aharmim, M. Auger et al., "Observation of two-neutrino double-beta decay in ${ }^{136} \mathrm{Xe}$ with the EXO-200 detector," Physical Review Letters, vol. 107, no. 21, Article ID 212501, 5 pages, 2011.

[6] A. Gando, Y. Gando, H. Hanakago et al., "Measurement of the double- $\beta$ decay half-life of ${ }^{136} \mathrm{Xe}$ with the KamLAND-Zen experiment," Physical Review C, vol. 85, no. 4, Article ID 045504, 6 pages, 2012.

[7] B. Schwingenheuer, "Status and prospects of searches for neutrinoless double beta decay," Annalen der Physik, vol. 525, no. 4, pp. 269-280, 2013.

[8] A. S. Barabash, "Experiment double beta decay: historical review of 75 years of research," Physics of Atomic Nuclei, vol. 74, no. 4, pp. 603-613, 2011.

[9] J. J. Gomez-Cadenas, J. Martin-Albo, M. Mezzetto, F. Monrabal, and M. Sorel, "The search for neutrinoless double beta decay," Rivista del Nuovo Cimento, vol. 35, no. 2, pp. 29-98, 2012.
[10] S. R. Elliott, "Recent progress in double beta decay," Modern Physics Letters, vol. 27, no. 7, Article ID 1230009, 2012.

[11] K.-H. Ackermann, M. Agostini, M. Allardt et al., "The Gerda experiment for the search of $0 \nu \beta \beta$ decay in ${ }^{76} \mathrm{Ge}$," The European Physical Journal C, vol. 73, p. 2330, 2013.

[12] D. G. Philips II, E. Aguayo, F. T. Avignone III et al., "The MAJORANA experiment: an ultra-low background search for neutrinoless double-beta decay," Journal of Physics: Conference Series, vol. 381, no. 1, Article ID 012044, 2012.

[13] P. Finnerty, E. Aguayo, M. Amman et al., "The MAJORANA DEMONSTRATOR: progress towards showing the feasibility of a tonne-scale ${ }^{76} \mathrm{Ge}$ neutrinoless double-beta decay experiment," in Proceedings of the 18th International Symposium on Particles, Strings and Cosmology, Merida, Mexico, June 2012.

[14] B. J. Mount, M. Redshaw, and E. G. Myers, "Double- $\beta$-decay Q values of ${ }^{74}$ Se and ${ }^{76} \mathrm{Ge}$," Physical Review $C$, vol. 81, no. 3, Article ID 032501, 4 pages, 2010.

[15] H. V. Klapdor-Kleingrothaus, A. Dietz, L. Baudis et al., "Latest results from the HEIDELBERG-MOSCOW double beta decay experiment," The European Physical Journal A, vol. 12, no. 2, pp. 147-154, 2001.

[16] C. E. Aalseth, F. T. Avignone, R. L. Brodzinski et al., "IGEX ${ }^{76} \mathrm{Ge}$ neutrinoless double-beta decay experiment: prospects for next generation experiments," Physical Review D, vol. 65, Article ID 092007, 6 pages, 2002.

[17] A. Morales, "Review on double beta decay experiments and comparison with theory," Nuclear Physics B, vol. 77, no. 1, pp. 335-345, 1999.

[18] A. Morales and J. Morales, "The neutrinoless double beta decay: the case for germanium detectors," Nuclear Physics B-Proceedings Supplements, vol. 114, pp. 141-157, 2003.

[19] H. V. Klapdor-Kleingrothaus, I. V. Krivosheina, A. Dietz, and O. Chkvorets, "Search for neutrinoless double beta decay with enriched ${ }^{76} \mathrm{Ge}$ in Gran Sasso 1990-2003," Physics Letters B, vol. 586, no. 3-4, pp. 198-212, 2004.

[20] H. V. Klapdor-Kleingrothaus and I. V. Krivosheina, "The evidence for the observation of $0 \nu \beta \beta$ decay: The identification of $0 v \beta \beta$ events from the full spectra," Modern Physics Letters A, vol. 21, no. 20, pp. 1547-1566, 2006.

[21] M. Auger, D. J. Auty, P. S. Barbeau et al., "Search for neutrinoless double-beta decay in ${ }^{136}$ Xe with EXO-200," Physical Review Letters, vol. 109, Article ID 032505, 6 pages, 2012.

[22] A. Gando, Y. Gando, H. Hanakago et al., "Limit on neutrinoless $\beta \beta$ decay of ${ }^{136} \mathrm{Xe}$ from the first phase of KamLAND-Zen and comparison with the positive claim in ${ }^{76} \mathrm{Ge}$," Physical Review Letters, vol. 110, Article ID 062502, 5 pages, 2013.

[23] S. Riboldi, C. A. Ur, M. Bellato et al., "A multi-channel digital acquisition system for Ge spectroscopy in the GERDA experiment," in Proceedings of the 18th IEEE Nuclear Science Symposium and International Workshop on Room Temperature Semiconductor Detectors, p. 1386, October 2010.

[24] M. Agostini, J. A. Detwiler, P. Finnerty et al., "The MGDO software library for data analysis in Ge neutrinoless double-beta decay experiments," Journal of Physics: Conference Series, vol. 375, Article ID 042027, 2012.

[25] R. Brun and F. Rademakers, "ROOT-an object oriented data analysis framework," Nuclear Instruments and Methods in Physics Research, Section A, vol. 389, no. 1-2, pp. 81-86, 1997.

[26] M. Agostini, L. Pandola, P. Zavarise, and O. Volynets, "GELATIO: a general framework for modular digital analysis of highpurity Ge detector signals," Journal of Instrumentation, vol. 6, Article ID P08013, 2011. 
[27] E. Andreotti, A. Garfagnini, W. Maneschg et al., "HEROICA: an underground facility for the fast screening of germanium detectors," Journal of Instrumentation, vol. 8, Article ID P06012, 2013.

[28] M. Agostini, M. Allardt, E. Andreotti et al., "The background in the neutrinoless double beta decay experiment Gerda," accepted by The European Physical Journal C, http://arxiv.org/ $\mathrm{abs} / 1306.5084$.

[29] Z. He, "Review of the Shockley-Ramo theorem and its application in semiconductor gamma-ray detectors," Nuclear Instruments and Methods in Physics Research Section A, vol. 463, p. 250, 2001.

[30] F. T. Avignone III, R. L. Brodzinski, J. I. Collar et al., "Theoretical and experimental investigation of cosmogenic radioisotope production in germanium," Nuclear Physics B-Proceedings Supplements, vol. 28A, no. 1, pp. 280-285, 1992.

[31] M. Agostini, M. Allardt, E. Andreotti et al., "Measurement of the half-life of the two-neutrino double beta decay of ${ }^{76} \mathrm{Ge}$ with the GERDA experiment," Journal of Physics G, vol. 40, no. 3, Article ID 035110, 2013.

[32] M. Agostini, M. Allardt, E. Andreotti et al., "Pulse shape discrimination for Gerda phase I data," The European Physical Journal C, vol. 73, p. 2583, 2013.

[33] M. Agostini, M. Allardt, E. Andreotti et al., "Results on neutrinoless double- $\beta$ decay of ${ }^{76} \mathrm{Ge}$ from phase I of the GERDA experiment," Physical Review Letters, vol. 111, no. 12, Article ID 122503, 6 pages.

[34] A. Rodin, A. Faessler, F. Šimkovic, and P. Vogel, "Assessment of uncertainties in QRPA $0 \nu \beta \beta$-decay nuclear matrix elements," Nuclear Physics A, vol. 766, pp. 107-131, 2006.

[35] A. Rodin, A. Faessler, F. Šimkovic, and P. Vogel, "Erratum to: "Assessment of uncertainties in QRPA $0 \nu \beta \beta$-decay nuclear matrix elements'”' Nuclear Physics A, vol. 793, no. 1-4, pp. 213-215, 2007.

[36] F. Simkovic, A. Faessler, V. Rodin, P. Vogel, and J. Engel, "Anatomy of the $0 \nu \beta \beta$ nuclear matrix elements," Physical Review $C$, vol. 77, no. 4, Article ID 045503, 11 pages, 2008.

[37] F. Šimkovic, R. Hodák, A. Faessler, and P. Vogel, "Relation between the $0 \nu \beta \beta$ and $2 \nu \beta \beta$ nuclear matrix elements reexamined," Physical Review C, vol. 83, Article ID 015502, 9 pages, 2011.

[38] E. Caurier, F. Nowacki, and A. Poves, "Shell Model description of the $\beta \beta$ decay of ${ }^{136} \mathrm{Xe}$, Physics Letters $B$, vol. 711, no. 1, pp. 62-64, 2012.

[39] J. Barea and F. Iachello, "Neutrinoless double- $\beta$ decay in the microscopic interacting boson model," Physical Review C, vol. 79, no. 4, Article ID 044301, 16 pages, 2009.

[40] J. Suhonen and O. Civitarese, "Double-beta-decay nuclear matrix elements in the QRPA framework," Journal of Physics G, vol. 39, no. 8, Article ID 085105, 2012.

[41] O. Civitarese and J. Suhonen, "Extracting information on the O $\nu \beta \beta$ decays from the $2 \nu \beta \beta$ decays," Nuclear Physics A, vol. 761, no. 3-4, pp. 313-332, 2005.

[42] E. W. Grewe, C. Bäumer, H. Dohmann et al., "The $\left(d,{ }^{2} \mathrm{He}\right)$ reaction on ${ }^{76} \mathrm{Se}$ and the double- $\beta$-decay matrix elements for $A=76$," Physical Review C, vol. 78, no. 4, Article ID 044301, 7 pages, 2008.

[43] J. H. Thies, D. Frekers, T. Adachi et al., "The $\left({ }^{3} \mathrm{He}, t\right)$ reaction on ${ }^{76} \mathrm{Ge}$, and the double- $\beta$-decay matrix element," Physical Review $C$, vol. 86, Article ID 014304, 11 pages, 2012.

[44] A. Caldwell and K. Kröninger, "Signal discovery in sparse spectra: a Bayesian analysis," Physical Review D, vol. 74, no. 9, Article ID 092003, 7 pages, 2006.
[45] M. Boswell, Y.-D. Chan, J. A. Detwiler et al., "MaGe-a Geant4based Monte Carlo application framework for low-background germanium experiments," IEEE Transactions on Nuclear Science, vol. 58, no. 3, pp. 1212-1220, 2011.

[46] S. Agostinelli, J. Allison, K. Amako et al., "Geant4-a simulation toolkit," Nuclear Instruments and Methods in Physics Research Section A, vol. 506, no. 3, pp. 250-303, 2003.

[47] J. Allison, K. Amako, J. Apostolakis et al., "Geant4 developments and applications," IEEE Transactions on Nuclear Science, vol. 53, no. 1, pp. 270-278, 2006.

[48] O. A. Ponkratenko, V. I. Tretyak, and Y. G. Zdesenko, "Event generator DECAY4 for simulating double-beta processes and decays of radioactive nuclei," Physics of Atomic Nuclei, vol. 63, no. 7, pp. 1282-1287, 2000.

[49] A. Caldwell, D. Kollar, and K. Kroninger, "BAT-the Bayesian analysis toolkit," Computer Physics Communications, vol. 180, no. 11, pp. 2197-2209, 2009.

[50] J. Kotila and F. Iachello, "Phase-space factors for double- $\beta$ decay," Physical Review C, vol. 85, no. 3, Article ID 034316, 2012.

[51] G. Meierhofer, P. Kudejova, L. Canella, P. Grabmayr, J. Jochum, and J. Jolie, "Thermal neutron capture cross-section of ${ }^{76} \mathrm{Ge}$," The European Physical Journal A, vol. 40, no. 1, pp. 61-64, 2009.

[52] L. Pandola, M. Bauer, K. Kröninger et al., "Monte Carlo evaluation of the muon-induced background in the GERDA double beta decay experiment," Nuclear Instruments and Methods in Physics Research Section A, vol. 570, pp. 149-158, 2007.

[53] D. Budjáš, M. B. Heider, O. Chkvorets, N. Khanbekov, and S. Schönert, "Pulse shape discrimination studies with a BroadEnergy Germanium detector for signal identification and background suppression in the GERDA double beta decay experiment," Journal of Instrumentation, vol. 4, no. 10, Article ID P10007, 2009.

[54] M. Agostini, C. A. Ur, D. Budjáš et al., "Signal modeling of high-purity Ge detectors with a small read-out electrode and application to neutrinoless double beta decay search in Ge-76," Journal of Instrumentation, vol. 6, Article ID P03005, 2011.

[55] A. A. Vasenko, I. V. Kirpichnikov, V. A. Kuznetsov et al., "New results in the ITEP/YePI double beta-decay experiment with enriched germanium detectors," Modern Physics Letters A, vol. 5, pp. 1299-1306, 1990.

[56] H. S. Miley, R. L. Brodzinski, J. H. Reeves, F. T. Avignone III, and J. I. Collar, "Suggestive evidence for the two-neutrino doublebeta decay of Ge-76," Physical Review Letters, vol. 65, p. 3092, 1990.

[57] F. T. Avignone, R. L. Brodzinski, C. K. Guerard et al., "Confirmation of the observation of $2 \nu \beta \beta$ decay of ${ }^{76} \mathrm{Ge}$," Physics Letters $B$, vol. 256, pp. 559-561, 1991.

[58] F. T. Avignone III, "Double-beta decay: somerecent results and developments," Progress in Particle and Nuclear Physics, vol. 32, pp. 223-245, 1994.

[59] M. Günther, J. Hellmig, G. Heusser et al., "Heidelberg-Moscow $\beta \beta$ experiment with ${ }^{76} \mathrm{Ge}$ : full setup with five detectors," Physical Review D, vol. 55, pp. 54-67, 1997.

[60] C. Dörr and H. V. Klapdor-Kleingrothaus, "New MonteCarlo simulation of the HEIDELBERG-MOSCOW double beta decay experiment," Nuclear Instruments and Methods in Physics Research, Section A, vol. 513, no. 3, pp. 596-621, 2003.

[61] A. M. Bakalyarov, A. Ya. Balysh, S. T. Belyaev, V. I. Lebedev, and S. V. Zhukov, "Results of experiment on investigation of ${ }^{76} \mathrm{Ge}$ double beta decay," Physics of Particles and Nuclei Letters, vol. 2, no. 2, pp. 77-81, 2005. 
[62] B. Pritychenko, in Proceedings of the Nuclear Structure, (East Lansing, Mi, June).

[63] TMVA, "Toolkit for Multivariate Data Analysis with Root," http://tmva.sourceforge.net/.

[64] P. S. Bhupal Dev, S. Goswami, M. Mitra, and W. Rodejohann, "Constraining neutrino mass from neutrinoless double beta decay," Physical Review D, vol. 88, no. 9, Article ID 091301(R), 6 pages, 2013. 

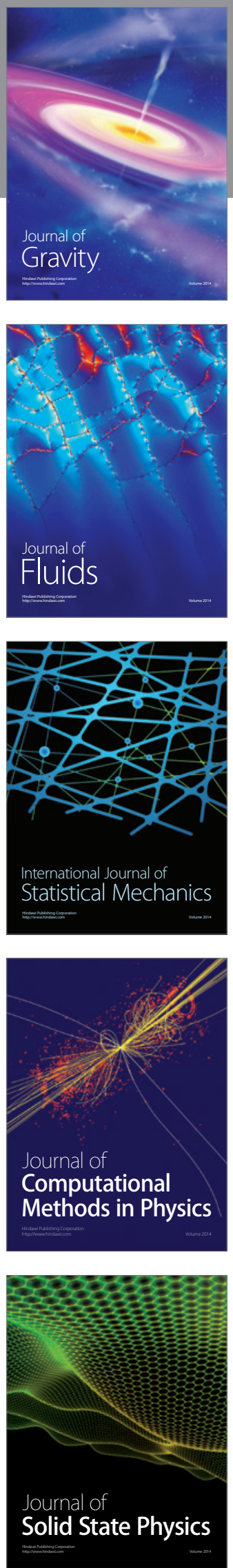

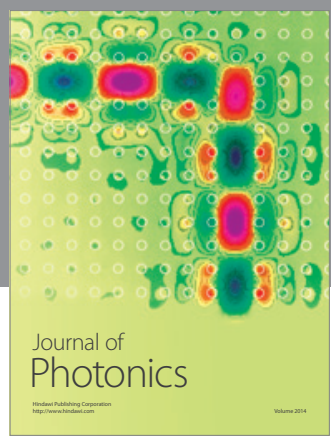

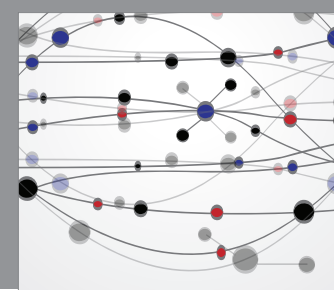

The Scientific World Journal

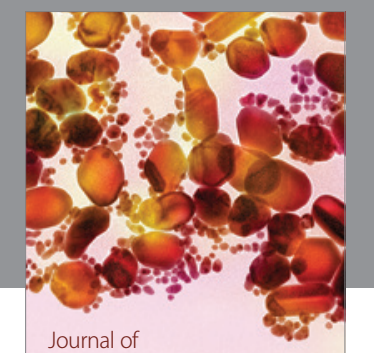

Soft Matter
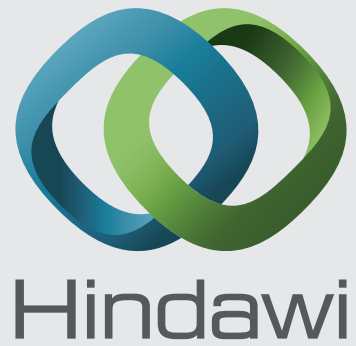

Submit your manuscripts at

http://www.hindawi.com
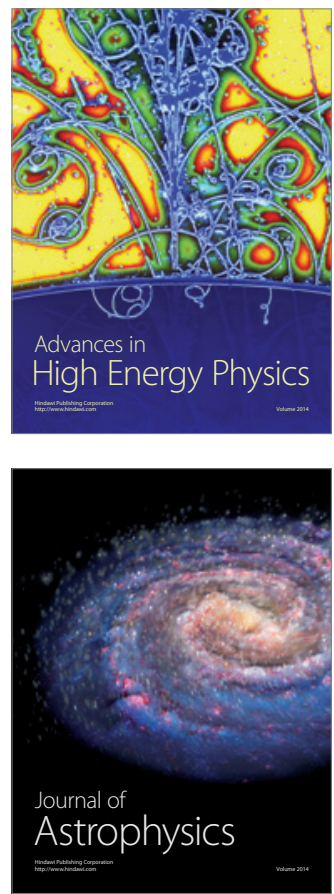
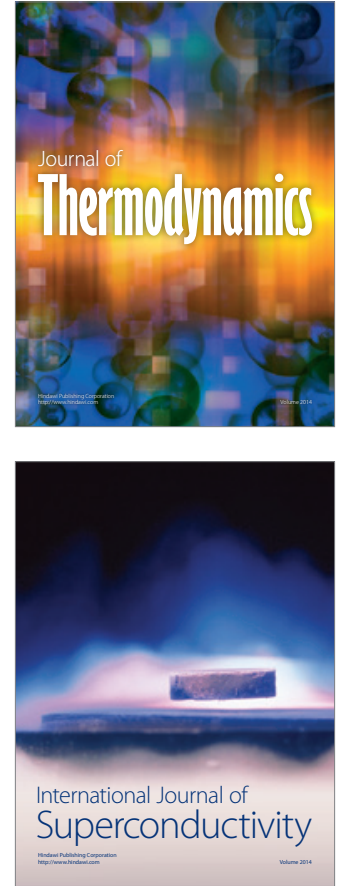
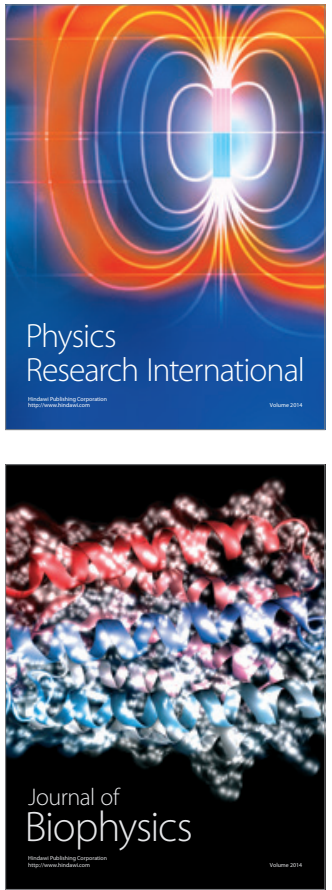
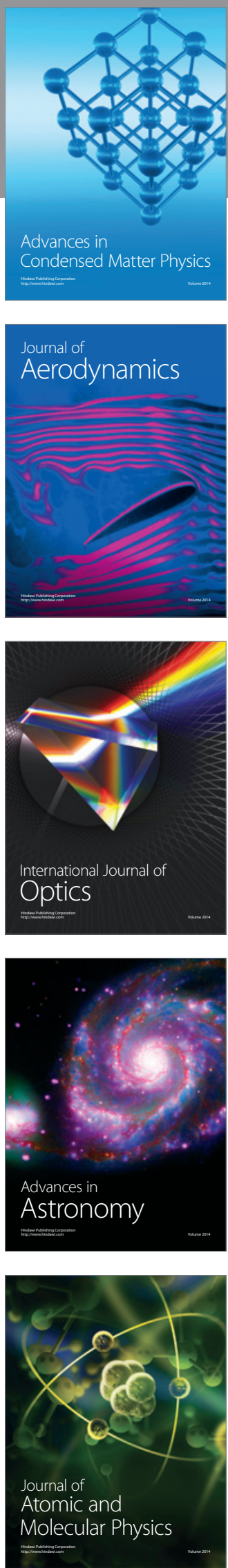